\title{
Survival impact of postoperative therapy modalities according to margin status in non-small cell lung cancer patients in the United States
}

\author{
Matthew P. Smeltzer, PhD, ${ }^{a}$ Chun Chieh Lin, MBA, PhD, ${ }^{b}$ Feng-Ming (Spring) Kong, MD, PhD, FACR,
} Ahmedin Jemal, DVM, PhD, ${ }^{b}$ and Raymond U. Osarogiagbon, MBBS, FACP ${ }^{\mathrm{d}}$

\begin{abstract}
Objective: Unlike complete (R0) resection guidelines, current National Comprehensive Cancer Network (NCCN) adjuvant therapy guidelines after incomplete (R1/R2) resection of non-small cell lung cancer (NSCLC) are based on low-level evidence. We attempted to validate them.

Methods: Patients with pathologic stage I-IIIA NSCLC from 2004 to 2011 in the National Cancer Database were stratified by margin status, NCCN-specified stage groupings, and adjuvant therapy exposure (none, radiotherapy, chemotherapy, or both). Five-year overall survival (OS) and hazard ratios, adjusted for patient and institutional characteristics, were compared. We used a parallel analysis of R0 resections to validate our methodology.
\end{abstract}

Results: We analyzed $3461 \mathrm{R} 1 / \mathrm{R} 2$, and 78,979 R0 resections. After R0 resection, the NCCN-recommended option was associated with the best survival across all stage groups, supporting our analytic approach. Patients with R1/R2 stage IA treated with radiation had a $26 \%$ OS, compared with $58 \%$ with no treatment $(P=.003)$. In patients with stage IB/IIA(N0) R1/R2, radiation was associated with a $25 \%$ OS compared with $47 \%$ with no treatment $(P=.025)$ and $62 \%$ with chemotherapy $(P<.007)$. Chemoradiation was not associated with a survival benefit in either group. Patients with IIA(N1)/IIB and IIIA had better survival with chemotherapy or chemoradiation. No group had a survival benefit with radiation alone.

Conclusions: NCCN adjuvant therapy guidelines after complete resection, based on high-level evidence, are validated, but not guidelines for patients with incompletely resected early-stage NSCLC, which are based on low-level evidence. Monomodality postoperative radiotherapy was not validated for any stage. Specific studies are needed to determine optimal management after incomplete resection. (J Thorac Cardiovasc Surg 2017;154:661-72)

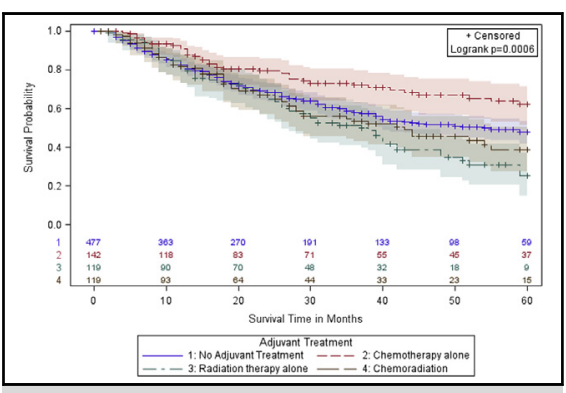

Kaplan-Meier survival curves for margin-positive patients in stage IB (T2a, N0) and stage IA (T2b, N0).

Central Message

NCCN guidelines for postoperative therapy after incomplete surgical resection in patients with stage I-II should be prospectively evaluated.

\section{Perspective}

NCCN guidelines for postoperative chemotherapy and radiation after complete surgical resection for NSCLC, based on high-level evidence, are validated in this analysis. Current guidelines for postoperative therapy after incomplete resection of stage I-II NSCLC, which are based on lower-level evidence, are not supported by this analysis.

See Editorial Commentary page 673.

See Editorial page 650 .

\footnotetext{
From the ${ }^{\mathrm{a} S c h o o l}$ of Public Health, University of Memphis; ${ }^{\mathrm{b}}$ American Cancer Society, Inc, Atlanta, Ga; ${ }^{\mathrm{c} D e p a r t m e n t}$ of Radiation Oncology, IU Simon Cancer Center, Indiana University School of Medicine, Indianapolis, Ind; and ${ }^{\mathrm{d}}$ Thoracic Oncology Research Group, Baptist Cancer Center, Memphis, Tenn.

Dr Osarogiagbon was partially supported by National Institutes of Health RO1CA172253 and Patient-Centered Outcomes Research Institute IH-13046147. Drs Jemal and Lin were supported by the American Cancer Society intramural research funding. Dr Kong was supported by National Institutes of Health R01CA142840, P01CA59827, and R01CA66948.

Received for publication July 27, 2016; revisions received March 1, 2017; accepted for publication March 19, 2017; available ahead of print May 5, 2017.

Address for reprints: Raymond U. Osarogiagbon, MBBS, FACP, Thoracic Oncology

Research Group, Baptist Cancer Center, 80 Humphreys Center Drive, Suite 220,

Memphis, TN 38120 (E-mail: rosarogi@ bmhcc.org).

$0022-5223 / \$ 36.00$

Copyright (c) 2017 by The American Association for Thoracic Surgery

http://dx.doi.org/10.1016/j.jtcvs.2017.03.085
}

Lung cancer accounts for approximately $27 \%$ of all annual US cancer deaths. ${ }^{1}$ Most long-term survivors are among the $29 \%$ of patients who have undergone curative-intent surgical resection. ${ }^{2,3}$ In high-risk patients, adjuvant chemotherapy ${ }^{4-6}$ and/or postoperative radiotherapy (PORT) may improve survival. ${ }^{7}$ The quality of evidence for the benefit of these treatments varies by stage and margin status. ${ }^{7-10}$

Scanning this QR code will take you to the supplemental tables and figures for this article.

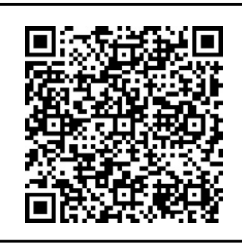




$$
\begin{aligned}
& \text { Abbreviations and Acronyms } \\
& \mathrm{HR}=\text { hazard ratio } \\
& \text { IQR = interquartile range } \\
& \text { NCCN }=\text { National Comprehensive Cancer } \\
& \text { Network } \\
& \mathrm{NCDB}=\text { National Cancer Database } \\
& \text { NSCLC }=\text { non }- \text { small cell lung cancer } \\
& \text { OS = overall survival } \\
& \text { PORT = postoperative radiotherapy } \\
& \text { R0 = complete resection (with uninvolved } \\
& \text { margins) } \\
& \mathrm{R} 1=\text { incomplete resection (with } \\
& \text { microscopically involved margins) } \\
& \text { R2 = incomplete resection (with } \\
& \text { macroscopically involved margins) } \\
& \mathrm{RCT}=\text { randomized clinical trials }
\end{aligned}
$$

Randomized clinical trials (RCTs) and a pooled analysis have demonstrated the benefit of adjuvant chemotherapy in completely (R0) resected patients with T-category $2 \mathrm{~b}$ or more advanced primary tumors, and those with nodal metastasis. $^{4-6,11}$ A large meta-analysis showed the harmfulness of PORT in R0-resected patients without mediastinal nodal metastasis ${ }^{12,13}$; a retrospective analysis of the US Surveillance, Epidemiology and End Results database and an unplanned retrospective analysis of a clinical trial suggest R0 patients with mediastinal nodal metastasis may benefit from PORT. ${ }^{7,10}$

Unlike the situation after complete resection, there is no RCT evidence to guide adjuvant management for the $2 \%$ to $17 \%$ of non-small cell lung cancer (NSCLC) resections with microscopic (R1)- or macroscopic (R2)-positive margins. ${ }^{14-16}$ However, recipients of incomplete resection are at significantly high risk for early death, irrespective of stage. ${ }^{16-18}$ Current National Comprehensive Cancer Network (NCCN) guideline recommendations for postoperative management of these patients are based on unverified expert opinion. ${ }^{19}$ Therefore, the guidelines need validation.

We evaluated the survival impact of 4 different adjuvant therapy options, after incomplete resection, in the National Cancer Database (NCDB) to determine which options seemed best for patients grouped into stage clusters as in the NCCN guidelines. ${ }^{19}$

\section{METHODS}

\section{Cohort Selection}

We used the NCDB, an oncology database sourced from Commission on Cancer-accredited Facilities, which covers approximately $70 \%$ of newly diagnosed US cancer cases. ${ }^{20,21}$ We selected patients with surgically resected pathologic stage I-IIIA NSCLC from 2004 to 2011 (International Classification of Disease for Oncology, 3rd version site codes C34.0-C34.9), excluding patients with missing information on last date of contact, administration (or date of administration) of radiation or chemotherapy, facility, or patient location. We also excluded patients with more than 1 surgical procedure, neoadjuvant radiation or chemotherapy, no (or unknown) nodal examination, adjuvant therapy more than 180 days past date of surgery, government insurance, and death within 60 days of surgery.

\section{Objectives}

The primary objective of this analysis was to compare stage-specific survival between postoperative therapy modalities in patients with incomplete surgical resection (R1/R2) who did not undergo re-resection. We used a parallel analysis of R0 patients to evaluate whether our data and methodology produced results congruent with existing high-level evidence for treatment of R0 patients.

\section{Adjuvant Therapy Options}

We classified postoperative therapy modalities as chemotherapy, radiotherapy, chemoradiation, or no treatment. Therapy administered within 6 months after surgery, at any dose level, was included as postoperative therapy. The median time from surgery to onset of treatment, by modality, is reported in Table E1. For combined-modality chemoradiation therapy, the second modality had to begin within 2 months of the end of the first. The time from surgery to initiation of adjuvant therapy was evaluated to verify that adjuvant modalities were not typically used for the purpose of salvage therapy in this cohort.

\section{NCCN Stage Groups and Adjuvant Therapy Guidelines}

NCCN recommendations for adjuvant therapy are based on pathologic stage, categorized into the following 4 groups: (1) stage IA (T1ab, N0); (2) stage IB (T2a, N0) and stage IIA (T2b, N0); (3) stage IIA (T1ab-T2a, N1) and stage IIB (T3, N0; T2b N1); and (4) stage IIIA (T1-3, N2; T3, $\mathrm{N} 1$ ). The NCCN-recommended nonsurgical adjuvant therapy for group 1 is PORT; for group 2, PORT with or without chemotherapy; for groups 3 and 4, chemoradiation (sequential or concurrent) for R1 and concurrent chemoradiation for R2. ${ }^{19}$

\section{Variables}

Margin status was evaluated as negative (R0) or positive (R1, R2, or positive not otherwise specified), and in subsequent analyses R1 and R2 were evaluated individually. Covariates (detailed in Table 1 and Table E2) in the analysis included patient demographics (age, sex, race, insurance status, income, rural/urban residence, census region), and clinical characteristics (comorbidity score $[0,1$, or $\geq 2]$, histology, tumor grade, tumor size, primary site, type of surgery), as well as institutional characteristics (facility type).

\section{Statistical Analysis}

Overall survival (OS) times were taken from the date of surgery until the date of death or last follow-up. Survival analyses were conducted to compare the 4 postoperative treatment modalities within each of the 4 stage groups. OS was estimated using the Kaplan-Meier method and postoperative treatment groups were compared using the log-rank test.

OS comparisons also were evaluated using univariate and multiple variable Cox proportional hazards models to adjust for covariates. Model-based hazard ratio estimates are reported with $95 \%$ confidence intervals. For each model, we present unadjusted hazard ratios and hazard ratios adjusted for demographic, clinical, surgical, and institutional characteristics. The proportional hazards assumption was evaluated graphically, using $\log (-\log )$ survival plots by treatment group. We used "no adjuvant treatment" as the reference adjuvant therapy option, because there is no clinical trial evidence to support adjuvant therapy after 
TABLE 1. Patient demographic and institutional characteristics among margin-positive patients by stage group

\begin{tabular}{|c|c|c|c|c|c|c|}
\hline Categories & $\begin{array}{c}\text { Total } \\
\mathbf{n}=\mathbf{3 4 6 1} \\
\mathbf{n}(\%)\end{array}$ & $\begin{array}{c}\text { Stage IA } \\
\text { (T1ab, N0) } \\
\mathbf{n}=\mathbf{3 6 9} \\
\mathbf{n}(\%)\end{array}$ & $\begin{array}{c}\text { Stage IB (T2a, N0) } \\
\text { and IIA (T2b, N0) } \\
\mathbf{n}=\mathbf{8 5 7} \\
\mathbf{n}(\%)\end{array}$ & $\begin{array}{c}\text { Stage IIA } \\
\text { (T1ab-T2a, N1) } \\
\text { and IIB } \\
\mathbf{n}=1317 \\
\mathbf{n}(\%)\end{array}$ & $\begin{array}{c}\text { Stage IIIA } \\
\text { (T1-T3, N2; T3, N1) } \\
\text { n }=918 \\
\text { n }(\%)\end{array}$ & $P$ \\
\hline \multicolumn{7}{|l|}{ Age group } \\
\hline $18-49$ & $227(7)$ & $16(4)$ & $40(5)$ & $90(7)$ & $81(9)$ & \multirow[t]{4}{*}{$<.0001$} \\
\hline $50-64$ & $1190(34)$ & $105(28)$ & 247 (29) & $476(36)$ & $362(39)$ & \\
\hline $65-74$ & $1230(36)$ & $141(38)$ & $328(38)$ & $458(35)$ & $303(33)$ & \\
\hline $75-90$ & $814(24)$ & 107 (29) & $242(28)$ & $293(22)$ & $172(19)$ & \\
\hline \multicolumn{7}{|l|}{ Sex } \\
\hline Male & $1851(53)$ & $159(43)$ & $436(51)$ & $766(58)$ & $490(53)$ & \multirow[t]{2}{*}{$<.0001$} \\
\hline Female & $1610(47)$ & $210(57)$ & $421(49)$ & $551(42)$ & $428(47)$ & \\
\hline \multicolumn{7}{|l|}{ Race/Ethnicity } \\
\hline Non-Hispanic, white & $2653(77)$ & $287(78)$ & $670(78)$ & $1000(76)$ & $696(76)$ & \multirow[t]{5}{*}{.20} \\
\hline Hispanic & $73(2)$ & $10(3)$ & $14(2)$ & $27(2)$ & $22(2)$ & \\
\hline Black & $342(10)$ & $39(11)$ & $79(9)$ & $123(9)$ & $101(11)$ & \\
\hline Other & $88(3)$ & $6(2)$ & $16(2)$ & $48(4)$ & $18(2)$ & \\
\hline Missing & $305(9)$ & $27(7)$ & $78(9)$ & $119(9)$ & $81(9)$ & \\
\hline \multicolumn{7}{|l|}{ Insurance } \\
\hline Uninsured & $87(3)$ & $6(2)$ & $19(2)$ & $40(3)$ & $22(2)$ & \multirow[t]{6}{*}{$<.0001$} \\
\hline Medicaid & $176(5)$ & $15(4)$ & $36(4)$ & $63(5)$ & $62(7)$ & \\
\hline Younger Medicare & $219(6)$ & $22(6)$ & $42(5)$ & $74(6)$ & $81(9)$ & \\
\hline Older Medicare & $1758(51)$ & $221(60)$ & $497(58)$ & $635(48)$ & $405(44)$ & \\
\hline Private & $1184(34)$ & $103(28)$ & $257(30)$ & $485(37)$ & $339(37)$ & \\
\hline Missing & $37(1)$ & $2(1)$ & $6(1)$ & $20(2)$ & $9(1)$ & \\
\hline \multicolumn{7}{|l|}{ Median income-quartile } \\
\hline$<\$ 30,000$ & $478(14)$ & $52(14)$ & $117(14)$ & $180(14)$ & $129(14)$ & \multirow[t]{5}{*}{.84} \\
\hline$\$ 30,000-\$ 34,999$ & $708(20)$ & $67(18)$ & $175(20)$ & $276(21)$ & $190(21)$ & \\
\hline$\$ 35,000-\$ 45,999$ & 999 (29) & $100(27)$ & $242(28)$ & $382(29)$ & $275(30)$ & \\
\hline$\$ 46,000+$ & $1095(32)$ & $126(34)$ & $275(32)$ & $407(31)$ & $287(31)$ & \\
\hline Missing & $181(5)$ & $24(7)$ & $48(6)$ & $72(5)$ & $37(4)$ & \\
\hline \multicolumn{7}{|l|}{ Comorbidity } \\
\hline 0 & $1611(47)$ & $144(39)$ & $387(45)$ & $623(47)$ & $457(50)$ & \multirow[t]{3}{*}{.014} \\
\hline 1 & $1270(37)$ & $149(40)$ & $321(37)$ & $491(37)$ & 309 (34) & \\
\hline $2+$ & $580(17)$ & $76(21)$ & $149(17)$ & $203(15)$ & $152(17)$ & \\
\hline \multicolumn{7}{|l|}{ Histology } \\
\hline Not otherwise specified & $9(0)$ & $1(0)$ & $1(0)$ & $7(1)$ & $0(0)$ & \multirow[t]{5}{*}{$<.0001$} \\
\hline Large cell & $177(5)$ & $14(4)$ & $34(4)$ & $70(5)$ & $59(6)$ & \\
\hline Squamous & $1340(39)$ & $121(33)$ & $335(39)$ & $572(43)$ & $312(34)$ & \\
\hline Other & $248(7)$ & $16(4)$ & $49(6)$ & $110(8)$ & $73(8)$ & \\
\hline Adenocarcinoma & $1687(49)$ & $217(59)$ & $438(51)$ & $558(42)$ & $474(52)$ & \\
\hline \multicolumn{7}{|l|}{ Tumor grade } \\
\hline Well/moderately differentiated & $1688(49)$ & $244(66)$ & $477(56)$ & $579(44)$ & $388(42)$ & \multirow[t]{3}{*}{$<.0001$} \\
\hline Poorly/undifferentiated & $1641(47)$ & $105(28)$ & $343(40)$ & $695(53)$ & $498(54)$ & \\
\hline Unknown & $132(4)$ & $20(5)$ & $37(4)$ & $43(3)$ & $32(3)$ & \\
\hline \multicolumn{7}{|l|}{ Tumor size, $\mathrm{cm}$} \\
\hline$\leq 3$ & $1339(39)$ & $356(96)$ & $279(33)$ & $418(32)$ & $286(31)$ & \multirow[t]{4}{*}{$<.0001$} \\
\hline$>3-\leq 5$ & $1156(33)$ & $3(1)$ & $367(43)$ & $457(35)$ & $329(36)$ & \\
\hline$>5$ & $940(27)$ & $8(2)$ & 207 (24) & $428(33)$ & $297(32)$ & \\
\hline Unknown & $26(1)$ & $2(1)$ & $4(0)$ & $14(1)$ & $6(1)$ & \\
\hline \multicolumn{7}{|l|}{ Rural/Urban } \\
\hline Rural & $664(19)$ & $65(18)$ & 165 (19) & $263(20)$ & $171(19)$ & \multirow[t]{3}{*}{.46} \\
\hline Urban & $2582(75)$ & $279(76)$ & $631(74)$ & $970(74)$ & $702(76)$ & \\
\hline Unknown & $215(6)$ & $25(7)$ & $61(7)$ & $84(6)$ & $45(5)$ & \\
\hline
\end{tabular}




\begin{tabular}{|c|c|c|c|c|c|c|}
\hline Categories & $\begin{array}{c}\text { Total } \\
\mathbf{n}=\mathbf{3 4 6 1} \\
\mathbf{n}(\%)\end{array}$ & $\begin{array}{c}\text { Stage IA } \\
\text { (T1ab, N0) } \\
\mathbf{n}=\mathbf{3 6 9} \\
\mathbf{n}(\%)\end{array}$ & $\begin{array}{c}\text { Stage IB (T2a, N0) } \\
\text { and IIA (T2b, N0) } \\
n=857 \\
n(\%)\end{array}$ & $\begin{array}{c}\text { Stage IIA } \\
\text { (T1ab-T2a, N1) } \\
\text { and IIB } \\
\mathbf{n}=1317 \\
\text { n }(\%)\end{array}$ & $\begin{array}{c}\text { Stage IIIA } \\
\text { (T1-T3, N2; T3, N1) } \\
\text { n = 918 } \\
\text { n }(\%)\end{array}$ & $\boldsymbol{P}$ \\
\hline \multicolumn{7}{|l|}{ Census region } \\
\hline Northeast & $572(17)$ & $72(20)$ & $136(16)$ & $207(16)$ & $157(17)$ & .58 \\
\hline Midwest & $1100(32)$ & $108(29)$ & $283(33)$ & $409(31)$ & $300(33)$ & \\
\hline South & $1366(39)$ & $150(41)$ & $327(38)$ & $532(40)$ & $357(39)$ & \\
\hline West & $423(12)$ & $39(11)$ & $111(13)$ & $169(13)$ & $104(11)$ & \\
\hline \multicolumn{7}{|l|}{ Primary site } \\
\hline C340-main bronchus & $54(2)$ & $1(0)$ & $13(2)$ & $15(1)$ & $25(3)$ & $<.0001$ \\
\hline C341-upper lobe & $2068(60)$ & $224(61)$ & $474(55)$ & $854(65)$ & $516(56)$ & \\
\hline C342-middle lobe & $174(5)$ & $22(6)$ & $54(6)$ & $46(3)$ & $52(6)$ & \\
\hline C343-lower lobe & $976(28)$ & $113(31)$ & $278(32)$ & $335(25)$ & $250(27)$ & \\
\hline C348-overlapping lesion & $124(4)$ & $3(1)$ & $28(3)$ & $40(3)$ & $53(6)$ & \\
\hline C349-lung not otherwise specified & $65(2)$ & $6(2)$ & $10(1)$ & $27(2)$ & $22(2)$ & \\
\hline \multicolumn{7}{|l|}{$\mathrm{T}$ category } \\
\hline $\mathrm{T} 1$ & $676(20)$ & $369(100)$ & $0(0)$ & $184(14)$ & $123(13)$ & $<.0001$ \\
\hline $\mathrm{T} 2$ & $1773(51)$ & $0(0)$ & $857(100)$ & $536(41)$ & $380(41)$ & \\
\hline $\mathrm{T} 3$ & $1012(29)$ & $0(0)$ & $0(0)$ & $597(45)$ & $415(45)$ & \\
\hline \multicolumn{7}{|l|}{$\mathrm{N}$ category } \\
\hline No & $1823(53)$ & $369(100)$ & $857(100)$ & $597(45)$ & $0(0)$ & $<.0001$ \\
\hline N1 & $964(28)$ & $0(0)$ & $0(0)$ & $720(55)$ & $244(27)$ & \\
\hline $\mathrm{N} 2$ & $674(19)$ & $0(0)$ & $0(0)$ & $0(0)$ & $674(73)$ & \\
\hline \multicolumn{7}{|l|}{ Surgery } \\
\hline Sublobar & $420(12)$ & $114(31)$ & $95(11)$ & $109(8)$ & $102(11)$ & $<.0001$ \\
\hline Lobe/bilobectomy & $2643(76)$ & $250(68)$ & $703(82)$ & $1060(80)$ & $630(69)$ & \\
\hline Pneumonectomy & $398(12)$ & $5(1)$ & $59(7)$ & $148(11)$ & $186(20)$ & \\
\hline \multicolumn{7}{|l|}{ Facility type } \\
\hline Community Cancer Program & $329(10)$ & $31(8)$ & $75(9)$ & $139(11)$ & $84(9)$ & .75 \\
\hline Comprehensive Community Cancer Program & $1772(51)$ & $191(52)$ & $445(52)$ & $679(52)$ & $457(50)$ & \\
\hline Teaching/Research Cancer Program & $738(21)$ & $79(21)$ & $188(22)$ & $274(21)$ & $197(22)$ & \\
\hline National Cancer Institute Program/Network & $299(9)$ & $39(11)$ & $72(8)$ & $104(8)$ & $84(9)$ & \\
\hline Other & $323(9)$ & $29(8)$ & $77(9)$ & $121(9)$ & $96(10)$ & \\
\hline
\end{tabular}

incomplete NSCLC resection. $P$ values less than .05 were considered statistically significant with no adjustment for multiple comparison and all analyses were conducted in SAS Version 9.4 (SAS Institute, Inc, Cary, NC). ${ }^{22}$

\section{Sensitivity Analyses}

We conducted multiple sensitivity analyses to address specific details of the analysis. First, the specific type of positive resection (R1 or R2) was unknown for some patients who were margin-positive. We evaluated the sensitivity of our results to margin-positivity of unknown type by conducting multiple analyses in which we grouped them as R1, R2, and eliminated them.

Additional sensitivity analyses were conducted to evaluate if departures from proportional hazards or the large number of covariates adjusted for in each model could affect the observed results from primary analysis. In these analyses, propensity score-adjusted models were used to control for demographic, clinical, surgical, and institutional characteristics with a propensity score, which was entered into the model as a covariate. ${ }^{23}$

Finally, we evaluated the potential impact of departures from the proportional hazards assumption by reevaluating the multiple variable Cox models after eliminating any exposure groups where the assumption was questionable.

\section{RESULTS}

A total of 82,440 patients were eligible: $3461(4 \%)$ with incomplete resection, the primary analysis group of interest (Figure 1), and 78,979 (96\%) with R0 resection (Figure E1), used to validate our analytic approach. The demographic and clinical characteristics of these patients, stratified by NCCN stage group (Table 1 [non-R0] and Table E2 [R0]) and adjuvant therapy exposure (Table 2 [non-R0] and Table E3 [R0]), are presented.

\section{Early-Stage Patients With Incomplete Resection: NCCN Groups 1 and 2}

OS estimates were compared by treatment modality in margin-positive patients with stage IA (T1ab, N0) and stages IB/IIA (T2a, N0 and T2b, N0). Margin-positive stage IA patients who received PORT alone had significantly lower OS compared with those with no treatment (5-year OS: $26 \%$ vs $58 \%, P=.0030$, Tables 3 


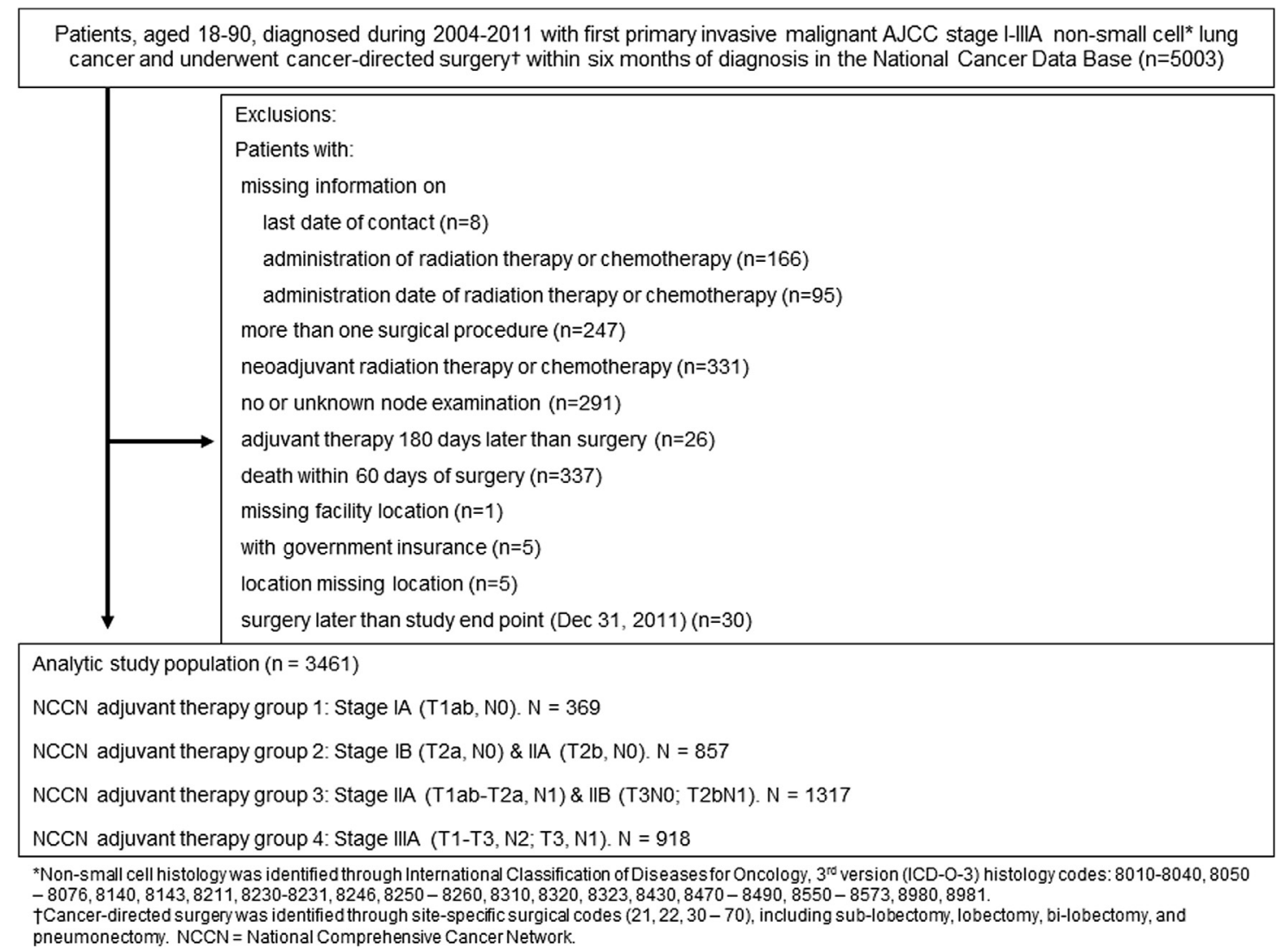

FIGURE 1. Study consort diagram for margin-positive patients. AJCC, American Joint Committee on Cancer.

and E4, Figure 2, A). This result trended toward statistical significance in the fully adjusted model (adjusted hazard ratio [aHR] 1.7, $P=.0551$, Table 3 ). Similarly, for patients with stage IB/IIA, the 5-year OS was $47 \%$ with no treatment, and $25 \%$ with PORT $(P=.0251$; aHR 1.28 , $P=.12$ ) (Table 3, Figure 2, $B$ ).

We found no significant association between chemotherapy and survival in patients with stage IA with positive margins; however, survival was significantly higher in persons with stages IB/IIA who received postoperative chemotherapy compared with no treatment (5-year OS: $62 \%$ vs $47 \%, P=.0065$, Table 3, Figure 2, $B$ ). These results remained statistically significant in fully adjusted models (aHR 0.58, $P=.0040$, Table 3 ). Sensitivity analysis using propensity score-adjusted models (Table E5), those that did not consider treatment groups in which the proportional hazards assumption may be violated (Table E6), and those including only anatomic resections (Table E7) provided consistent results. Survival with chemoradiation was not significantly different from no adjuvant treatment in group 1 or 2 patients (Table 3). Analysis restricted to patients with anatomic resections provided similar results (Table E7).

\section{Late-Stage Patients With Incomplete Resection: NCCN Groups 3 and 4}

In margin-positive NCCN group 3, patients with stage IIA (T1ab-T2a, N1) or stage IIB (T3, N0; T2b N1), those who received radiation had a similar survival experience to those who received no treatment (5-year OS: $26 \%$ vs $27 \%$, $P=.59$, Figure 2, $C$, Table 3). Recipients of chemotherapy or chemoradiation had superior survival $(P<.0010$, Table 3 , Figure $2, C$ ). Results were similar in fully adjusted models, where the chemotherapy group had 0.72 times the hazard of death compared with no treatment $(P=.0041)$, and the chemoradiation group had 0.74 times the hazard of death $(P=.0083)$.

Subsequent analysis found no substantial differences in survival in the chemoradiation group based on the order in which therapies were administered (Table E8). When evaluated separately, patients receiving chemotherapy first and then radiation had 37\% 5-year OS compared with $36 \%$ for patients receiving radiation first and then chemotherapy and $38 \%$ for those receiving both concurrently (Table E8).

Consistent with NCCN guidelines, margin-positive patients with stage IIA or stage IIB were further delineated 
TABLE 2. Patient demographic and institutional characteristics among margin-positive patients by adjuvant therapy

\begin{tabular}{|c|c|c|c|c|c|c|}
\hline Categories & $\begin{array}{c}\text { Total } \\
\mathbf{n}=\mathbf{3 4 6 1} \\
\mathbf{n}(\%) \\
\end{array}$ & $\begin{array}{c}\text { No treatment } \\
\mathbf{n}=1406 \\
\mathbf{n}(\%) \\
\end{array}$ & $\begin{array}{c}\text { Chemotherapy } \\
\begin{array}{c}n=645 \\
n(\%)\end{array} \\
\end{array}$ & $\begin{array}{c}\text { Radiation therapy } \\
n=447 \\
n(\%)\end{array}$ & $\begin{array}{c}\text { Chemoradiation } \\
\mathbf{n}=\mathbf{9 6 3} \\
\mathbf{n}(\%) \\
\end{array}$ & $P$ \\
\hline \multicolumn{7}{|l|}{ Stage group } \\
\hline Stage IA & $369(11)$ & 265 (19) & $19(3)$ & $60(13)$ & $25(3)$ & \multirow[t]{4}{*}{$<.0001$} \\
\hline Stage IB and IIA & $857(25)$ & 477 (34) & $142(22)$ & $119(27)$ & $119(12)$ & \\
\hline Stage IIA and IIB & $1317(38)$ & $419(30)$ & $284(44)$ & $199(45)$ & $415(43)$ & \\
\hline Stage IIIA & $918(27)$ & 245 (17) & $200(31)$ & $69(15)$ & $404(42)$ & \\
\hline \multicolumn{7}{|l|}{ Age group } \\
\hline 18-49 & $227(7)$ & $66(5)$ & $44(7)$ & $14(3)$ & $103(11)$ & \multirow[t]{4}{*}{$<.0001$} \\
\hline $50-64$ & $1190(34)$ & $390(28)$ & $271(42)$ & $106(24)$ & $423(44)$ & \\
\hline $65-74$ & $1230(36)$ & $508(36)$ & $227(35)$ & $170(38)$ & $325(34)$ & \\
\hline $75-90$ & $814(24)$ & $442(31)$ & $103(16)$ & $157(35)$ & $112(12)$ & \\
\hline \multicolumn{7}{|l|}{ Sex } \\
\hline Male & $1851(53)$ & $735(52)$ & $337(52)$ & $240(54)$ & $539(56)$ & \multirow[t]{2}{*}{.31} \\
\hline Female & $1610(47)$ & $671(48)$ & $308(48)$ & $207(46)$ & $424(44)$ & \\
\hline \multicolumn{7}{|l|}{ Race/Ethnicity } \\
\hline Non-Hispanic, white & $2653(77)$ & $1087(77)$ & $475(74)$ & $350(78)$ & $741(77)$ & \multirow[t]{5}{*}{.18} \\
\hline Hispanic & $73(2)$ & $35(2)$ & $15(2)$ & $8(2)$ & $15(2)$ & \\
\hline Black & $342(10)$ & $147(10)$ & $67(10)$ & $34(8)$ & $94(10)$ & \\
\hline Other & $88(3)$ & $29(2)$ & $18(3)$ & $17(4)$ & $24(2)$ & \\
\hline Missing & $305(9)$ & $108(8)$ & $70(11)$ & $38(9)$ & $89(9)$ & \\
\hline \multicolumn{7}{|l|}{ Insurance } \\
\hline Uninsured & $87(3)$ & $37(3)$ & $14(2)$ & $9(2)$ & $27(3)$ & \multirow[t]{6}{*}{$<.0001$} \\
\hline Medicaid & $176(5)$ & $69(5)$ & $23(4)$ & $23(5)$ & $61(6)$ & \\
\hline Younger Medicare & $219(6)$ & $77(5)$ & $44(7)$ & $16(4)$ & $82(9)$ & \\
\hline Older Medicare & $1758(51)$ & $823(59)$ & $275(43)$ & $296(66)$ & $364(38)$ & \\
\hline Private & $1184(34)$ & $388(28)$ & $281(44)$ & $96(21)$ & 419 (44) & \\
\hline Missing & $37(1)$ & $12(1)$ & $8(1)$ & $7(2)$ & $10(1)$ & \\
\hline \multicolumn{7}{|l|}{ Median income-quartile } \\
\hline$<\$ 30,000$ & $478(14)$ & $196(14)$ & $79(12)$ & $72(16)$ & $131(14)$ & \multirow[t]{5}{*}{.25} \\
\hline$\$ 30,000-\$ 34,999$ & $708(20)$ & $281(20)$ & $139(22)$ & $81(18)$ & 207 (22) & \\
\hline$\$ 35,000-\$ 45,999$ & $999(29)$ & 405 (29) & $171(27)$ & $132(30)$ & $291(30)$ & \\
\hline$\$ 46,000+$ & $1095(32)$ & $448(32)$ & $211(33)$ & $139(31)$ & $297(31)$ & \\
\hline Missing & $181(5)$ & $76(5)$ & $45(7)$ & $23(5)$ & $37(4)$ & \\
\hline \multicolumn{7}{|l|}{ Comorbidity } \\
\hline 0 & $1611(47)$ & $634(45)$ & $331(51)$ & $183(41)$ & $463(48)$ & \multirow[t]{3}{*}{.005} \\
\hline 1 & $1270(37)$ & $511(36)$ & $225(35)$ & $179(40)$ & $355(37)$ & \\
\hline $2+$ & $580(17)$ & $261(19)$ & 89 (14) & 85 (19) & $145(15)$ & \\
\hline \multicolumn{7}{|l|}{ Histology } \\
\hline Not otherwise specified & $9(0)$ & $3(0)$ & $2(0)$ & $1(0)$ & $3(0)$ & \multirow{5}{*}{$<.0001$} \\
\hline Large cell & $177(5)$ & $57(4)$ & $38(6)$ & $23(5)$ & $59(6)$ & \\
\hline Squamous & $1340(39)$ & $524(37)$ & $214(33)$ & $216(48)$ & $386(40)$ & \\
\hline Other & $248(7)$ & $90(6)$ & $46(7)$ & $32(7)$ & $80(8)$ & \\
\hline Adenocarcinoma & $1687(49)$ & $732(52)$ & $345(53)$ & $175(39)$ & $435(45)$ & \\
\hline \multicolumn{7}{|l|}{ Tumor grade } \\
\hline Well/moderately differentiated & $1688(49)$ & $753(54)$ & $307(48)$ & $208(47)$ & $420(44)$ & \multirow{3}{*}{$<.0001$} \\
\hline Poorly/undifferentiated & $1641(47)$ & $590(42)$ & $312(48)$ & $221(49)$ & $518(54)$ & \\
\hline Unknown & $132(4)$ & $63(4)$ & $26(4)$ & $18(4)$ & $25(3)$ & \\
\hline \multicolumn{7}{|l|}{ Tumor size, $\mathrm{cm}$} \\
\hline$\leq 3$ & 1339 (39) & $654(47)$ & $212(33)$ & $172(38)$ & $301(31)$ & \multirow{4}{*}{$<.0001$} \\
\hline$>3-\leq 5$ & $1156(33)$ & 417 (30) & $223(35)$ & $161(36)$ & 355 (37) & \\
\hline$>5$ & $940(27)$ & $323(23)$ & 207 (32) & $112(25)$ & $298(31)$ & \\
\hline Unknown & $26(1)$ & $12(1)$ & $3(0)$ & $2(0)$ & $9(1)$ & \\
\hline
\end{tabular}


TABLE 2. Continued

\begin{tabular}{|c|c|c|c|c|c|c|}
\hline Categories & $\begin{array}{c}\text { Total } \\
\mathrm{n}=\mathbf{3 4 6 1} \\
\mathbf{n}(\%)\end{array}$ & $\begin{array}{c}\text { No treatment } \\
\text { n }=1406 \\
n(\%)\end{array}$ & $\begin{array}{l}\text { Chemotherapy } \\
\begin{array}{c}n=645 \\
n(\%)\end{array}\end{array}$ & $\begin{array}{c}\text { Radiation therapy } \\
\qquad \begin{array}{c}n=47 \\
n(\%)\end{array}\end{array}$ & $\begin{array}{l}\text { Chemoradiation } \\
\begin{array}{c}\mathbf{n}=963 \\
\mathbf{n}(\%)\end{array}\end{array}$ & $P$ \\
\hline \multicolumn{7}{|l|}{ Rural/Urban } \\
\hline Rural & $664(19)$ & $285(20)$ & $110(17)$ & $90(20)$ & $179(19)$ & .014 \\
\hline Urban & $2582(75)$ & $1023(73)$ & $482(75)$ & $337(75)$ & $740(77)$ & \\
\hline Unknown & $215(6)$ & $98(7)$ & $53(8)$ & $20(4)$ & $44(5)$ & \\
\hline \multicolumn{7}{|l|}{ Census region } \\
\hline Northeast & $572(17)$ & $234(17)$ & $106(16)$ & $79(18)$ & $153(16)$ & $<.0001$ \\
\hline Midwest & $1100(32)$ & $386(27)$ & $222(34)$ & $144(32)$ & $348(36)$ & \\
\hline South & $1366(39)$ & $586(42)$ & $246(38)$ & $158(35)$ & $376(39)$ & \\
\hline West & $423(12)$ & $200(14)$ & $71(11)$ & $66(15)$ & $86(9)$ & \\
\hline \multicolumn{7}{|l|}{ Primary site } \\
\hline C340-main bronchus & $54(2)$ & $16(1)$ & $8(1)$ & $12(3)$ & $18(2)$ & .22 \\
\hline C341-upper lobe & $2068(60)$ & 825 (59) & $384(60)$ & $280(63)$ & $579(60)$ & \\
\hline C342-middle lobe & $174(5)$ & $76(5)$ & $34(5)$ & $14(3)$ & $50(5)$ & \\
\hline C343-lower lobe & $976(28)$ & $412(29)$ & $185(29)$ & $122(27)$ & $257(27)$ & \\
\hline C348-overlapping lesion & $124(4)$ & $45(3)$ & $22(3)$ & $16(4)$ & $41(4)$ & \\
\hline C349-lung not otherwise specified & $65(2)$ & $32(2)$ & $12(2)$ & $3(1)$ & $18(2)$ & \\
\hline \multicolumn{7}{|l|}{$\mathrm{T}$ category } \\
\hline $\mathrm{T} 1$ & $676(20)$ & $368(26)$ & $82(13)$ & $89(20)$ & $137(14)$ & $<.0001$ \\
\hline $\mathrm{T} 2$ & $1773(51)$ & $757(54)$ & $388(60)$ & $191(43)$ & $437(45)$ & \\
\hline $\mathrm{T} 3$ & $1012(29)$ & $281(20)$ & $175(27)$ & $167(37)$ & $389(40)$ & \\
\hline \multicolumn{7}{|l|}{$\mathrm{N}$ category } \\
\hline No & $1823(53)$ & $912(65)$ & $247(38)$ & $309(69)$ & $355(37)$ & $<.0001$ \\
\hline N1 & $964(28)$ & $320(23)$ & $248(38)$ & $94(21)$ & $302(31)$ & \\
\hline $\mathrm{N} 2$ & $674(19)$ & $174(12)$ & $150(23)$ & $44(10)$ & $306(32)$ & \\
\hline \multicolumn{7}{|l|}{ Surgery } \\
\hline Sublobar & $420(12)$ & $180(13)$ & $56(9)$ & $78(18)$ & $106(11)$ & $<.0001$ \\
\hline Lobe/bilobectomy & $2643(76)$ & 1077 (77) & $482(75)$ & $335(75)$ & 749 (78) & \\
\hline Pneumonectomy & $398(12)$ & $149(11)$ & 107 (17) & $34(8)$ & $108(11)$ & \\
\hline \multicolumn{7}{|l|}{ Facility type } \\
\hline Community Cancer Program & $329(10)$ & $123(9)$ & $55(9)$ & $33(7)$ & $118(12)$ & $<.0001$ \\
\hline Comprehensive Community Cancer Program & $1772(51)$ & $687(49)$ & $328(51)$ & $249(56)$ & $508(53)$ & \\
\hline Teaching/Research Cancer Program & $738(21)$ & $343(24)$ & $131(20)$ & $97(22)$ & $167(17)$ & \\
\hline National Cancer Institute Program/Network & $299(9)$ & $133(9)$ & $66(10)$ & $32(7)$ & $68(7)$ & \\
\hline Other & $323(9)$ & $120(9)$ & $65(10)$ & $36(8)$ & $102(11)$ & \\
\hline
\end{tabular}

based on the specific type of incomplete resection, R1, R2, or unknown (margin-positive, but type not specified). Although potentially limited by smaller sample sizes, results were largely consistent with those observed for all margin-positive patients combined (Table E9).

In margin-positive NCCN group 4, patients with stage IIIA (T1-3, N2; T3, N1), 5-year OS was similar between patients who received PORT $(10 \%)$ and no treatment $(12 \%, P=.52$, Figure $2, D)$; however, compared with no treatment, patients with chemotherapy alone had higher 5 -year OS (21\% vs $12 \%, P=.0048)$, as did those with chemoradiation $(25 \%, P<.0001)$. Fully adjusted models confirmed these findings (Table 3 ). Specifically, the patients had a lower hazard of death in both the chemotherapy group (aHR 0.77, $P=.0466$ ) and the chemoradiation group (aHR $0.63, P<.0001)$, compared with no treatment. Analyses restricted to patients with anatomic resection provided similar results (Table E7).

Analysis of margin-positive patients with stage IIIA, after further stratification into R1 or R2 subsets, yielded similar results to the combined cohort (Table E9). Similar to group 3 patients, we found no meaningful difference in survival in patients with stage IIIA based on the order that chemoradiation was received (Table E8).

\section{Validation Analysis With Margin-Negative \\ Resections}

We applied the same analysis to the R0 resection cohort in a parallel analysis. Five-year OS, unadjusted proportional hazards models, and adjusted proportional hazards models in this cohort are presented in Table 4, Figure E2, and 
TABLE 3. Kaplan-Meier survival analysis and proportional hazards models by stage group for margin-positive patients

\begin{tabular}{|c|c|c|c|c|c|c|}
\hline & \multirow[b]{2}{*}{ Postoperative treatment } & \multicolumn{5}{|c|}{ Margin positive } \\
\hline & & $\mathbf{n}$ & $\begin{array}{c}5 \text {-y overall survival (\%) } \\
\text { (log-rank } P \text { value } \%)\end{array}$ & $\begin{array}{c}\text { Unadjusted hazard ratio } \\
(\mathbf{9 5} \% \mathbf{C I})\end{array}$ & $\begin{array}{c}\text { Adjusted hazard ratio } \dagger \\
\text { (95\% CI) }\end{array}$ & $P^{*}$ \\
\hline \multirow[t]{4}{*}{ Group 1: stage IA (T1ab, N0) } & No treatment & 265 & 58 (Referent) & 1.00 (Referent) & 1.00 (Referent) & \\
\hline & Chemo only & 19 & $65(.6687)$ & $0.81(0.35-1.86)$ & $1.27(0.48-3.38)$ & .6369 \\
\hline & Radiation only & 60 & $26(.0030)$ & $1.97(1.25-3.11)$ & $1.68(0.99-2.84)$ & .0551 \\
\hline & Chemo + Rad & 25 & $35(.0895)$ & $1.69(0.92-3.12)$ & $0.96(0.47-1.98)$ & .9176 \\
\hline \multirow{4}{*}{$\begin{array}{l}\text { Group 2: stage IB (T2a, N0) } \\
\text { and stage IIA (T2b, N0) }\end{array}$} & No treatment & 477 & 47 (Referent) & 1.00 (Referent) & 1.00 (Referent) & \\
\hline & Chemo only & 142 & $62(.0065)$ & $0.61(0.43-0.87)$ & $0.58(0.40-0.84)$ & .0040 \\
\hline & Radiation only & 119 & $25(.0251)$ & $1.39(1.04-1.86)$ & $1.28(0.94-1.74)$ & .1185 \\
\hline & Chemo + Rad & 119 & $39(.3571)$ & $1.15(0.85-1.57)$ & $0.97(0.70-1.35)$ & .8678 \\
\hline \multirow{4}{*}{$\begin{array}{l}\text { Group 3: stage IIA (T1ab- } \\
\text { T2a, N1) and stage IIB (T3, } \\
\text { N0; T2b N1) }\end{array}$} & No treatment & 419 & 27 (Referent) & 1.00 (Referent) & 1.00 (Referent) & \\
\hline & Chemo only & 284 & $36(.0001)$ & $0.65(0.53-0.81)$ & $0.72(0.58-0.90)$ & .0041 \\
\hline & Radiation only & 199 & $26(.5907)$ & $1.06(0.85-1.32)$ & $0.94(0.74-1.18)$ & .5878 \\
\hline & Chemo + Rad & 415 & $37(<.0001)$ & $0.68(0.56-0.83)$ & $0.76(0.62-0.93)$ & .0083 \\
\hline \multirow{4}{*}{$\begin{array}{l}\text { Group 4: stage IIIA (T1-3, } \\
\text { N2; T3, N1) }\end{array}$} & No treatment & 245 & 12 (Referent) & 1.00 (Referent) & 1.00 (Referent) & \\
\hline & Chemo only & 200 & $21(.0048)$ & $0.70(0.55-0.90)$ & $0.77(0.60-1.00)$ & .0466 \\
\hline & Radiation only & 69 & $10(.5215)$ & $1.11(0.81-1.51)$ & $1.03(0.74-1.43)$ & .8729 \\
\hline & Chemo + Rad & 404 & $25(<.0001)$ & $0.59(0.48-0.72)$ & $0.63(0.51-0.79)$ & $<.0001$ \\
\hline
\end{tabular}

$C I$, Confidence interval; Chemo, chemotherapy; Rad, radiation. * $P$ values compare each treatment to referent (no treatment). $\dagger$ Adjusted for age, sex, race/ethnicity, insurance, median income, comorbidity, histology, tumor grade, tumor size, rural/urban, census region, primary site, $\mathrm{T}$ category, $\mathrm{N}$ category, surgery facility type, facility surgical $\%$ lung cancer, facility \% Medicaid or uninsured.

Table E10. We further delineated the stage IIIA marginnegative survival analysis by pN-category (N0/N1 vs N2) to match the NCCN guideline subsets and evaluated their comparative OS based on adjuvant therapy exposure (Table E11). The pattern of adjuvant therapy benefit in our analysis matched up with the evidence-based NCCN guidelines for $\mathrm{R} 0$ resection (Table 5).

\section{Comparison With NCCN Recommendations}

Results from margin-positive and margin-negative analyses by stage groups are summarized qualitatively in Table 5, and are compared with the current NCCN recommendations.

\section{DISCUSSION}

We compared OS between adjuvant therapy modalities in patients with completely and incompletely resected NSCLC, to determine if current NCCN recommendations are supported by a robust nationally representative dataset. Our primary interest was in the patients with incomplete resection, but we used the $\mathrm{R} 0$ cohort to validate our methodology, and the suitability of the NCDB for this purpose. This analysis consistently corroborated NCCN guidelines backed by high-level clinical trial evidence, but did not support current recommendations in several scenarios after incomplete resection, where the available evidence is sparse.

In patients with completely resected stage IA NSCLC, RCTs have shown no benefit from adjuvant therapy. ${ }^{4,6}$ In stage IB-IIB, RCTs and a pooled analysis including the 5 largest studies, have shown an increase in overall and relapse-free survival with postoperative cisplatin-based chemotherapy compared with observation. ${ }^{4-6,11}$ Our analysis of the R0 cohort is consistent with this evidence. Specifically, patients with completely resected stage IB-IIA NSCLC who received chemotherapy had results superior to all other treatment groups. In patients with completely resected stage IIIA NSCLC, current evidence supports chemotherapy for those with N0 or N1, and chemotherapy or chemoradiation for those with $\mathrm{N} 2$, which is the current NCCN recommendation. ${ }^{19}$ The R0 cohort analysis supports the use of chemotherapy in patients with $\mathrm{N} 0$ and N1, and chemotherapy with or without radiation in patients with $\mathrm{N} 2$.

Incomplete resections occur relatively infrequently, and adjuvant therapy trials specifically exclude these patients. $^{4-7,10,11,24}$ Therefore, there is no definitive evidence on the best choice of postoperative therapy in this situation. ${ }^{16-18}$ NCCN guidelines currently recommend PORT for group 1 (stage IA), PORT with or without chemotherapy for group 2 (stage IB and IIA), and chemoradiation for groups 3 (stage IIA with N1 and IIB) and 4 (T3N1 and T1-3,N2). ${ }^{19}$ Our analysis supports observation for group 1, chemotherapy only for group 2 , chemotherapy with or without radiation for group 3 , and chemoradiation therapy for group 4 . This analysis supports the NCCN recommendations for groups 3 and 4, but suggests that the current recommendations may be harmful to patients in groups 1 and 2 . It also does not support the use of PORT alone in any subset.

Recent publications using the NCDB have provided conflicting results on the value of PORT after incomplete resection. Hancock and colleagues ${ }^{18}$ found that chemotherapy or chemotherapy plus PORT provided superior 

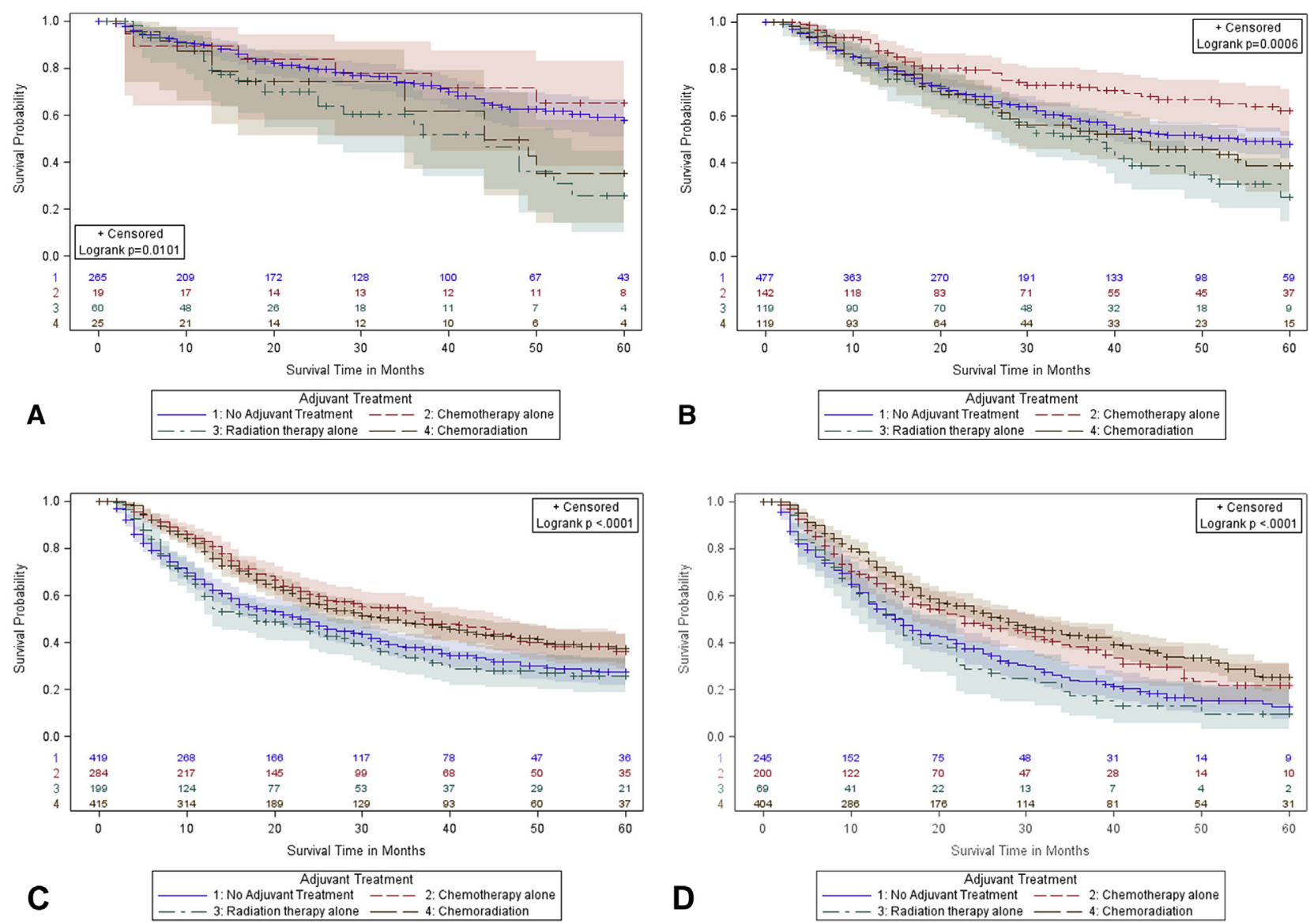

FIGURE 2. Kaplan-Meier survival curves for margin-positive patients categorized by National Comprehensive Cancer Network adjuvant therapy stage groups. The log-rank $P$ value tests the null hypothesis that all 4 groups have similar survival. A, Group 1-stage IA (T1ab, N0). B, Group 2-stage IB (T2a, N0) and stage IIA (T2b, N0). C, Group 3-stage IIA (T1ab-T2a, N1) and stage IIB (T3, N0; T2b N1). D, Group 4- stage IIIA (T1-3, N2; T3, N1).

results for stages I-III. However patients who received PORT alone after incomplete resection had unimproved (stage II-III) or worse (stage I) survival. Wang and colleagues $^{25}$ reported slightly longer survival in patients completing a full regime of PORT at 50 to 74 Gy postoperatively. Key differences in our study may explain the conflicting results.

Our analysis of the NCDB used the NCCN adjuvant therapy stage groupings in an attempt to validate the treatment guidelines. Therefore, we further delineated stage I patients by pT-category and stage IIA patients by pN-category. This delineation, coupled with the broader timeline (2004-2011 vs 2003-2006), may explain the subtle difference between our findings and those of Hancock and colleagues. ${ }^{18}$ Both studies found that early-stage patients receiving PORT alone had shorter survival. However, we found the best survival for early-stage (NCCN group 2) patients was with chemotherapy alone compared with the findings of Hancock and colleagues, ${ }^{18}$ who found that chemotherapy with or without PORT both showed similar survival that was superior to no adjuvant treatment or PORT alone for the undilineated group of patients with stage II and III.

The report by Wang and colleagues, ${ }^{25}$ supporting the use of PORT in patients with stage II-III with incomplete resection, differed from our work by evaluating only patients with an optimal PORT experience. Specifically, Wang and colleagues ${ }^{25}$ excluded all patients who died within 120 days of surgery, and included only patients who completed optimal-dose radiation. A less optimal classification of PORT use is more pragmatic and provides better information for treatment of patients, whose ability to receive a full treatment regimen of PORT cannot be known at the time of treatment decision. Patients who died as a result of acute radiation complications would have been excluded from their analysis. Another difference is that they treated chemotherapy as a confounding variable rather than a separate treatment option as we, and Hancock and colleagues, ${ }^{18}$ have done. 
TABLE 4. Kaplan-Meier survival analysis and proportional hazards models by stage group for margin-negative patients

\begin{tabular}{|c|c|c|c|c|c|c|}
\hline & \multirow[b]{2}{*}{$\begin{array}{c}\text { Postoperative } \\
\text { treatment }\end{array}$} & \multicolumn{5}{|c|}{ Margin negative } \\
\hline & & $\mathbf{n}$ & $\begin{array}{c}\text { 5-y overall } \\
\text { survival }(\%) \\
(\log -\text { rank } P \text { value } *)\end{array}$ & $\begin{array}{c}\text { Unadjusted } \\
\text { hazard ratio } \\
(95 \% \mathrm{CI})\end{array}$ & $\begin{array}{c}\text { Adjusted } \\
\text { hazard ratio } \dagger \\
(\mathbf{9 5} \% \mathbf{C I})\end{array}$ & $\boldsymbol{P}^{*}$ \\
\hline \multirow[t]{4}{*}{ Group 1: stage IA (T1ab, N0) } & No treatment & 33,780 & 71 (Referent) & 1.00 (Referent) & 1.00 (Referent) & \\
\hline & Chemo only & 789 & $74(.2946)$ & $0.92(0.78-1.08)$ & $1.02(0.87-1.20)$ & .8160 \\
\hline & Radiation only & 136 & $44(<.0001)$ & $2.89(2.22-3.73)$ & $2.18(1.67-2.85)$ & $<.0001$ \\
\hline & Chemo + Rad & 76 & $40(<.0001)$ & $3.19(2.21-4.59)$ & $2.99(2.07-4.31)$ & $<.0001$ \\
\hline \multirow{4}{*}{$\begin{array}{l}\text { Group 2: stage IB (T2a, N0) } \\
\text { and stage IIA (T2b, N0) }\end{array}$} & No treatment & 19,281 & 57 (Referent) & 1.00 (Referent) & 1.00 (Referent) & \\
\hline & Chemo only & 4568 & $68(<.0001)$ & $0.68(0.63-0.72)$ & $0.74(0.69-0.80)$ & $<.0001$ \\
\hline & Radiation only & 250 & $38(<.0001)$ & $1.92(1.60-2.31)$ & $1.80(1.49-2.16)$ & $<.0001$ \\
\hline & Chemo + Rad & 215 & $47(.0003)$ & $1.47(1.19-1.81)$ & $1.41(1.14-1.74)$ & .0016 \\
\hline \multirow{4}{*}{$\begin{array}{l}\text { Group 3: stage IIA (T1ab- } \\
\text { T2a, N1) endstage IIB (T3, } \\
\text { N0; T2b N1) }\end{array}$} & No treatment & 6101 & 37 (Referent) & 1.00 (Referent) & 1.00 (Referent) & \\
\hline & Chemo only & 5788 & $53(<.0001)$ & $0.59(0.56-0.63)$ & $0.66(0.62-0.70)$ & $<.0001$ \\
\hline & Radiation only & 354 & $28(<.0001)$ & $1.35(1.17-1.57)$ & $1.36(1.18-1.58)$ & $<.0001$ \\
\hline & Chemo + Rad & 895 & $40(.1772)$ & $0.93(0.84-1.03)$ & $1.04(0.93-1.16)$ & .4811 \\
\hline \multirow{4}{*}{$\begin{array}{l}\text { Group 4: stage IIIA (T1-3, } \\
\text { N2; T3, N1) }\end{array}$} & No treatment & 2119 & 24 (Referent) & 1.00 (Referent) & 1.00 (Referent) & \\
\hline & Chemo only & 2520 & $39(<.0001)$ & $0.59(0.55-0.65)$ & $0.63(0.58-0.69)$ & $<.0001$ \\
\hline & Radiation only & 248 & $18(.0747)$ & $1.18(0.99-1.39)$ & $1.15(0.97-1.37)$ & .1025 \\
\hline & Chemo + Rad & 1859 & $38(<.0001)$ & $0.62(0.57-0.68)$ & $0.69(0.63-0.76)$ & $<.0001$ \\
\hline
\end{tabular}

$\overline{C I}$, Confidence interval; Chemo, chemotherapy; Rad, radiation. ${ }^{*} P$ values compare each treatment to referent (no treatment). $\dagger$ Adjusted for age, sex, race/ethnicity, insurance, median income, comorbidity, histology, tumor grade, tumor size, rural/urban, census region, primary site, $\mathrm{T}$ category, $\mathrm{N}$ category, surgery facility type, facility surgical $\%$ lung cancer, facility \% Medicaid or uninsured.

Our PORT analysis group included all persons who survived 60 days after surgery and received treatment with PORT within 6 months of surgery. Patients who discontinued PORT or who received PORT at a less-thanoptimal dose were included to adhere to the intention-totreat principle and avoid potential selection bias. Because treatment with PORT alone may be carried out differently from PORT with chemotherapy, we considered these 2 treatment options separately to better represent clinical practice and to avoid the potential for residual confounding by controlling for chemotherapy use exclusively through statistical modeling.
This retrospective study has several limitations. We have expressly excluded the primary recommendation of re-resection for non-R0 resections because of the relatively small number of such patients in the database. Ideally, PORT is preferably commenced within 60 days. We used a 6-month eligibility window, as others have done in these types of analyses, to reflect the practical reality that some patients start adjuvant therapy late. ${ }^{18,25}$ The median time to onset of PORT alone was 52 days, and $75 \%$ of patients initiated therapy within 74 days. This suggests that PORT was used adjuvantly, and not for salvage therapy after disease progression. However,

TABLE 5. Comparative survival impact of adjuvant therapy in patients with completely and incompletely resected stage I-IIIA non-small cell lung cancer in the NCDB, current NCCN adjuvant therapy recommendations, and objective results from our analysis

\begin{tabular}{|c|c|c|c|c|c|c|}
\hline Stage & Margin status & Chemo & Radiation & Chemo + RT & NCCN & NCDB data \\
\hline \multirow[t]{2}{*}{ Group 1: stage IA } & Negative & Neutral & Worse & Worse & Observe & Supports observation \\
\hline & Positive & Neutral & Worse & Neutral & Radiation & Supports observation \\
\hline \multirow[t]{2}{*}{ Group 2: stage IB and stage IIA } & Negative & Better & Worse & Worse & Observe or Chemo & Supports chemo only \\
\hline & Positive & Better & Neutral & Neutral & $\mathrm{RT} \pm$ Chemo & Supports chemo only \\
\hline \multirow[t]{4}{*}{ Group 3: stage IIA and stage IIB } & Negative & Better & Worse & Neutral & Chemo & Supports chemo only \\
\hline & Positive & Better & Neutral & Better & & \\
\hline & $\mathrm{R} 1$ & Better & Neutral & Better & Chemo + RT & Supports chemo \pm RT \\
\hline & $\mathrm{R} 2$ & \multicolumn{3}{|c|}{ Insufficient data (Table E9) } & Chemo + RT & \\
\hline \multirow[t]{3}{*}{ Group 4: stage IIIA } & Negative & Better & Neutral & Better & & \\
\hline & Non-N2 & Better & Neutral & Neutral & Chemo & Supports chemo only \\
\hline & N2 & Better & Worse & Better & Chemo + RT & Supports chemo $\pm \mathrm{RT}$ \\
\hline \multirow[t]{3}{*}{ Group 4: stage IIIA } & Positive & Better & Neutral & Better & & \\
\hline & $\mathrm{R} 1$ & Neutral & Neutral & Better & Chemo + RT & Supports chemo + RT \\
\hline & $\mathrm{R} 2$ & \multicolumn{5}{|c|}{ Insufficient data (Table E9) } \\
\hline
\end{tabular}

$N C D B$, National Cancer Database; $N C C N$, National Comprehensive Cancer Network; Chemo, chemotherapy; $R T$, radiation therapy; Chemo $+R T$, combined-modality chemotherapy and radiation therapy administered sequentially. 
it is impossible to verify the clinical circumstances around any of the treatments.

The NCDB covers $70 \%$ of all lung cancer cases in the United States, drawing from a diverse group of hospitals; however, results may not apply directly to substantially different institutions. Although the NCDB is thorough, incomplete and inaccurate data are still potential problems. Although we addressed this limitation for critical variables by validating our results with sensitivity analyses, unequal assignment of postoperative treatment modalities may have affected our results and the sample size of some analysis subsets may be too small for meaningful statistical inference. Outside a well-executed RCT, this remains a potential explanation for differences observed in all studies of this question. We have addressed this limitation as well as possible, with extensive adjustment by statistical analysis.

The lack of observed benefit from PORT or chemoradiation in early-stage patients after incomplete resection parallels the current evidence in completely resected patients; the impact of radiation therapy in reducing the increased cancer-related mortality risk after incomplete resection does not seem to overcome the excessive treatment-related mortality risk of PORT. ${ }^{26}$ Chemotherapy appears to be valuable to some degree across stage groups; patients with mediastinal nodal metastasis seem to benefit from chemotherapy or combined-modality chemoradiation.

Well-conducted retrospective evaluations can lead to conflicting conclusions based on selection criteria for assigning treatment groups after the fact. An inherent imbalance between treatment groups before treatment initiation is likely when treatment is selected based on physician decision after individual patient assessment. Statistical adjustment is unlikely to completely eliminate such confounding-by-indication.

This study provides the most comprehensive evaluation of NCCN guidelines for postoperative therapy to date. Results are largely consistent with high-level evidence available after complete surgical resection. In patients with incomplete resection, in whom the available evidence is far less, these data did not support the use of PORT in early-stage patients. All available evidence in incompletely resected patients is lower-level, and results are discrepant. Only RCTs can definitively determine the best adjuvant therapy for incompletely resected NSCLC.

Such a trial will be challenging to execute because of the relatively low incidence of incomplete resections, and the practical reality that incomplete resections are least frequent in the types of institutions that typically conduct clinical trials. ${ }^{16}$ However, infrastructure such as the National Cancer Institute's Community Oncology Research Program can be harnessed to support such a trial. The possibility of patient harm in the existing evidence void should stimulate the political will to resolve this question.

\section{Conflict of Interest Statement}

Dr Osarogiagbon has served as a consultant for Roche/ Genentech and speaker for Pfizer and Roche/Genentech. Dr Kong has received research funding and travel support and a speaker's honorarium from Varian Medical System. All other authors have nothing to disclose with regard to commercial support.

This study used the National Cancer Database. The authors acknowledge the efforts of the American College of Surgeons, the Commission on Cancer, and the American Cancer Society in the creation of the National Cancer Database. All statements in this report, including its findings and conclusions, are solely those of the authors and do not necessarily represent the views of the American College of Surgeons, the Commission on Cancer, the American Cancer Society, and the Patent-Centered Outcomes Research Institute, its board of governors, or its methodology committee. Dr Chun Chieh Lin had full access to all of the data in the study and takes responsibility for the integrity of the data and the accuracy of the data analysis.

\section{References}

1. Siegel R, Ma J, Zou Z, Jemal A. Cancer statistics, 2014. CA Cancer J Clin. 2014; 64:9-29.

2. Pfannschmidt J, Muley T, Bulzebruck H, Hoffmann H, Dienemann H. Prognostic assessment after surgical resection for non-small cell lung cancer: experiences in 2083 patients. Lung cancer. 2007;55:371-7.

3. Little AG, Rusch VW, Bonner JA, Gaspar LE, Green MR, Webb WR, et al. Patterns of surgical care of lung cancer patients. Ann Thorac Surg. 2005;80: 2051-6; discussion 2056.

4. Arriagada R, Bergman B, Dunant A, Le Chevalier T, Pignon JP, Vansteenkiste J. Cisplatin-based adjuvant chemotherapy in patients with completely resected non-small-cell lung cancer. N Engl J Med. 2004;350:351-60.

5. Winton T, Livingston R, Johnson D, Rigas J, Johnston M, Butts C, et al. Vinorelbine plus cisplatin vs. observation in resected non-small-cell lung cancer. $N$ Engl J Med. 2005;352:2589-97.

6. Pignon JP, Tribodet H, Scagliotti GV, Douillard JY, Shepherd FA, Stephens RJ, et al. Lung adjuvant cisplatin evaluation: a pooled analysis by the LACE Collaborative Group. J Clin Oncol. 2008;26:3552-9.

7. Lally BE, Zelterman D, Colasanto JM, Haffty BG, Detterbeck FC, Wilson LD. Postoperative radiotherapy for stage II or III non-small-cell lung cancer using the surveillance, epidemiology, and end results database. J Clin Oncol. 2006; 24:2998-3006

8. Keller SM, Adak S, Wagner H, Herskovic A, Komaki R, Brooks BJ, et al. A randomized trial of postoperative adjuvant therapy in patients with completely resected stage II or IIIA non-small-cell lung cancer. Eastern Cooperative Oncology Group. N Engl J Med. 2000;343: $1217-22$.

9. Weisenburger TH. Effects of postoperative mediastinal radiation on completely resected stage II and stage III epidermoid cancer of the lung. LCSG 773. Chest 1994; 106:297S-301S.

10. Douillard JY, Rosell R, De Lena M, Riggi M, Hurteloup P, Mahe MA. Impact of postoperative radiation therapy on survival in patients with complete resection and stage I, II, or IIIA non-small-cell lung cancer treated with adjuvant chemotherapy: the adjuvant Navelbine International Trialist Association (ANITA) Randomized Trial. Int J Radiat Oncol Biol Phys. 2008; 72:695-701.

11. Butts CA, Ding K, Seymour L, Twumasi-Ankrah P, Graham B, Gandara D, et al. Randomized phase III trial of vinorelbine plus cisplatin compared with observation in completely resected stage IB and II non-small-cell lung cancer: updated survival analysis of JBR-10. J Clin Oncol. 2010;28: 29-34.

12. Postoperative radiotherapy in non-small-cell lung cancer: systematic review and meta-analysis of individual patient data from nine randomised controlled trials. PORT Meta-analysis Trialists Group. Lancet. 1998;352: 257-63. 
13. Burdett S, Stewart L. PORT Meta-analysis Group. Postoperative radiotherapy in non-small-cell lung cancer: update of an individual patient data meta-analysis. Lung Cancer. 2005;47:81-3.

14. Lequaglie C, Conti B, Brega Massone PP, Giudice G. Unsuspected residual disease at the resection margin after surgery for lung cancer: fate of patients after long-term follow up. Eur J Cardiothorac Surg. 2003;23:229-32.

15. Kayser K, Anyanwu E, Bauer HG, Vogt-Moykopf I. Tumor presence at resection boundaries and lymph-node metastasis in bronchial carcinoma patients. Thorac Cardiovasc Surg. 1993;41:308-11.

16. Osarogiagbon RU, Lin CC, Smeltzer MP, Jemal A. Prevalence, prognostic implications, and survival modulators of incompletely resected non-small cell lung cancer in the U.S. National Cancer Data Base. J Thorac Oncol. 2016;11:e5-16.

17. Wind J, Smit EJ, Senan S, Eerenberg JP. Residual disease at the bronchial stump after curative resection for lung cancer. Eur J Cardiothorac Surg. 2007;32:29-34.

18. Hancock JG, Rosen JE, Antonicelli A, Moreno A, Kim AW, Detterbeck FC, et al. Impact of adjuvant treatment for microscopic residual disease after non-small cell lung cancer surgery. Ann Thorac Surg. 2015;99:406-13.

19. National Comprehensive Cancer Network. Non-Small Cell Lung Cancer NCCN guidelines Version 2. 2015. Available at: www.nccn.org/professionals/physician gls/pdf/nscl.pdf. Accessed December 16, 2014.

20. American College of Surgeons. Programs: National Cancer Data Base. 2011. Available at: http://www.facs.org/cancer/ncdb/index.html. Accessed December $16,2014$.
21. Lerro CC, Robbins AS, Phillips JL, Stewart AK. Comparison of cases captured in the national cancer data base with those in population-based central cancer registries. Ann Surg Oncol. 2013;20:1759-65.

22. Rothman KJ. No adjustments are needed for multiple comparisons. Epidemiology. 1990;1:43-6.

23. Austin P. An introduction to propensity score methods for reducing the effects of confounding in observational studies. Multivariate Behav Res. 2011;46:399-424.

24. The Lung Cancer Study Group. Effects of postoperative mediastinal radiation on completely resected stage II and stage III epidermoid cancer of the lung. $N$ Engl J Med. 1986;315:1377-81.

25. Wang EH, Corso CD, Rutter CE, Park HS, Chen AB, Kim AW, et al. Postoperative radiation therapy is associated with improved overall survival in incompletely resected stage II and III non-small-cell lung cancer. J Clin Oncol. 2015;33:2727-34.

26. Lally BE, Detterbeck FC, Geiger AM, Thomas CR Jr, Machtay M, Miller AA, et al. The risk of death from heart disease in patients with nonsmall cell lung cancer who receive postoperative radiotherapy: analysis of the Surveillance, Epidemiology, and End Results database. Cancer. 2007;110:911-7.

Key Words: incomplete resection, lung cancer, postoperative therapy, survival, National Comprehensive Cancer Network (NCCN), adjuvant therapy 
Patients, aged 18-90, diagnosed during 2004-2011 with first primary invasive malignant AJCC stage I-IIIA non-small cell lung cancer and underwent cancer-directed surgeryt within six months of diagnosis in the National Cancer Data Base $(n=101,381)$

\begin{tabular}{|c|c|}
\hline & 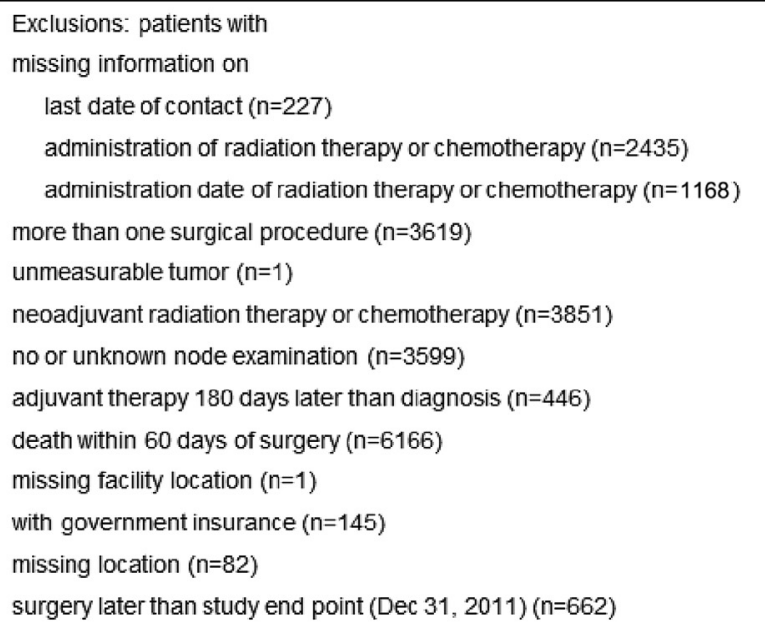 \\
\hline \multicolumn{2}{|c|}{ Analytic study population $(n=78979)$} \\
\hline \multicolumn{2}{|c|}{ NCCN Adjuvant therapy group 1: Stage IA (T1ab, N0) $N=34781$} \\
\hline \multicolumn{2}{|c|}{ NCCN Adjuvant therapy group 2: Stage IB (T2a, N0) \& $\|$ A (T2b, N0). N = 24314} \\
\hline \multicolumn{2}{|c|}{ NCCN Adjuvant therapy group 3: Stage IIA (T1ab-T2a, N1) \& IB (T3,NO; T2b,N1). N = 13138} \\
\hline \multicolumn{2}{|c|}{ NCCN Adjuvant therapy group 4: Stage IIIA (T1-T3, N2; T3, N1). N = 6746} \\
\hline
\end{tabular}

*Non-small cell histologywas identified through International Classification of Diseasesfor Oncology, $3^{\text {rd }}$ version (ICD-O-3) histology codes: 8010-8040, 8050 $-8076,8140,8143,8211,8230-8231,8246,8250-8260,8310,8320,8323,8430,8470-8490,8550-8573,8980,8981$.

†Cancer-directed surgery was identified through site-specific surgical codes $(21,22,30-70)$, including sub-lobectomy, labectomy, bi-lobectomy, and pneumonectomy. $\mathrm{NCCN}=$ National Comprehensive Cancer Network.

FIGURE E1. Consort diagram for margin-negative patients with non-small cell lung cancer (NSCLC). AJCC, American Joint Committee on Cancer. 

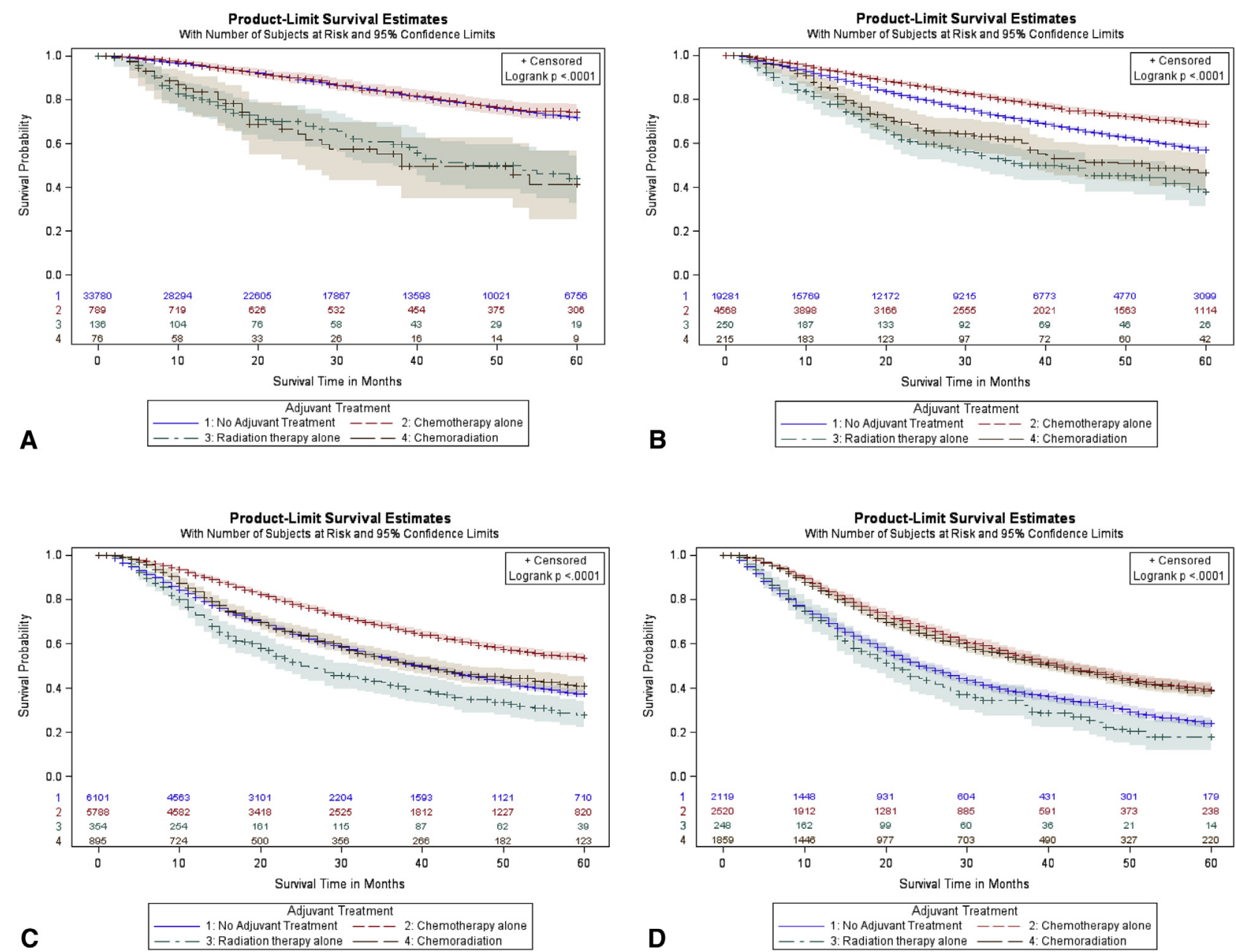

FIGURE E2. Kaplan-Meier survival curves for margin-negative patients by stage group. A, Group 1. B, Group 2. C, Group 3. D, Group 4. 
TABLE E1. Times from surgery to adjuvant therapy

\begin{tabular}{lrr}
\hline & Median & IQR \\
\hline Days from surgery to initiation of PORT in patients without chemotherapy & 52 & $39-74$ \\
Days from surgery to initiation of chemotherapy in patients without PORT & 47 & $35-62$ \\
In patients receiving chemotherapy and PORT & 57 & $40-126$ \\
Days from surgery to initiation of radiation therapy & 43 & $34-58$ \\
Days from surgery to initiation of chemotherapy & \\
\hline
\end{tabular}

$I Q R$, Interquartile range; $P O R T$, postoperative radiation therapy.

TABLE E2. Patient demographic and institutional characteristics among margin-negative patients by stage group

\begin{tabular}{|c|c|c|c|c|c|c|}
\hline Categories & $\begin{array}{c}\text { Total } \\
\mathbf{n}=\mathbf{7 8 , 9 7 9} \\
\mathbf{n}(\%)\end{array}$ & $\begin{array}{c}\text { Stage IA } \\
\text { (T1ab, N0) } \\
\begin{array}{c}\mathbf{n}=\mathbf{3 4 , 7 8 1} \\
\mathrm{n}(\%)\end{array}\end{array}$ & $\begin{array}{c}\text { Stage IB } \\
\text { (T2a, N0) and } \\
\text { IIA (T2b, N0) } \\
\mathbf{n}=\mathbf{2 4 , 3 1 4} \\
\mathbf{n}(\%)\end{array}$ & $\begin{array}{c}\text { Stage IIA } \\
\text { (T1ab-T2a, N1) } \\
\text { and IIB } \\
\text { n }=13,138 \\
\text { n }(\%)\end{array}$ & $\begin{array}{c}\text { Stage IIIA } \\
\text { (T1-T3,N2; T3, N1) } \\
\text { n }=6746 \\
\text { n }(\%)\end{array}$ & $\boldsymbol{P}$ \\
\hline \multicolumn{7}{|l|}{ Age group } \\
\hline $18-49$ & $4389(6)$ & $1870(5)$ & $1181(5)$ & $820(6)$ & $518(8)$ & \multirow[t]{4}{*}{$<.0001$} \\
\hline $50-64$ & $25,864(33)$ & $11,380(33)$ & $7352(30)$ & $4651(35)$ & $2481(37)$ & \\
\hline $65-74$ & $29,782(38)$ & $13,462(39)$ & 9084 (37) & $4871(37)$ & $2365(35)$ & \\
\hline $75-90$ & $18,944(24)$ & 8069 (23) & $6697(28)$ & $2796(21)$ & $1382(21)$ & \\
\hline \multicolumn{7}{|l|}{ Sex } \\
\hline Male & $37,844(48)$ & $14,933(43)$ & $12,480(51)$ & $7055(54)$ & $3376(50)$ & \multirow[t]{2}{*}{$<.0001$} \\
\hline Female & $41,135(52)$ & $19,848(57)$ & $11,834(49)$ & $6083(46)$ & $3370(50)$ & \\
\hline \multicolumn{7}{|l|}{ Race/Ethnicity } \\
\hline Non-Hispanic, white & $61,957(79)$ & $27,532(79)$ & $18,978(78)$ & $10,303(78)$ & $5144(76)$ & \multirow[t]{5}{*}{$<.0001$} \\
\hline Hispanic & $1734(2)$ & $709(2)$ & $565(2)$ & $278(2)$ & $182(3)$ & \\
\hline Black & $6478(8)$ & $2665(8)$ & $2029(8)$ & $1124(9)$ & $660(10)$ & \\
\hline Other & $2078(3)$ & 879 (3) & $657(3)$ & $322(3)$ & $220(3)$ & \\
\hline Missing & $6732(9)$ & $2996(9)$ & $2085(9)$ & $1111(9)$ & $540(8)$ & \\
\hline \multicolumn{7}{|l|}{ Insurance } \\
\hline Uninsured & $1517(2)$ & $563(2)$ & $493(2)$ & $286(2)$ & $175(3)$ & \multirow[t]{6}{*}{$<.0001$} \\
\hline Medicaid & $3325(4)$ & $1301(4)$ & $1030(4)$ & $635(5)$ & $359(5)$ & \\
\hline Younger Medicare & $4538(6)$ & $2120(6)$ & $1278(5)$ & $745(6)$ & $395(6)$ & \\
\hline Older Medicare & $41,134(52)$ & $18,298(53)$ & $13,253(55)$ & $6452(49)$ & $3131(46)$ & \\
\hline Private & $27,402(35)$ & $12,033(35)$ & 7946 (33) & $4828(37)$ & $2595(39)$ & \\
\hline Missing & $1063(1)$ & $466(1)$ & $314(1)$ & $192(2)$ & $91(1)$ & \\
\hline \multicolumn{7}{|l|}{ Median income-quartile } \\
\hline$<\$ 30,000$ & $10,308(13)$ & $4326(12)$ & $3287(14)$ & $1810(14)$ & $885(13)$ & \multirow[t]{5}{*}{$<.0001$} \\
\hline$\$ 30,000-\$ 34,999$ & $14,718(19)$ & $6277(18)$ & 4619 (19) & $2574(20)$ & $1248(19)$ & \\
\hline$\$ 35,000-\$ 45,999$ & $21,718(28)$ & $9454(27)$ & $6705(28)$ & $3723(28)$ & $1836(27)$ & \\
\hline$\$ 46,000+$ & $27,950(35)$ & $12,742(37)$ & $8414(35)$ & $4397(34)$ & $2397(36)$ & \\
\hline Missing & $4285(5)$ & $1982(6)$ & $1289(5)$ & $634(5)$ & $380(6)$ & \\
\hline \multicolumn{7}{|l|}{ Comorbidity } \\
\hline 0 & $37,470(47)$ & $16,095(46)$ & $11,671(48)$ & $6293(48)$ & $3411(51)$ & \multirow[t]{3}{*}{$<.0001$} \\
\hline 1 & $28,532(36)$ & $12,865(37)$ & $8532(35)$ & $4818(37)$ & $2317(34)$ & \\
\hline $2+$ & $12,977(16)$ & $5821(17)$ & $4111(17)$ & $2027(15)$ & $1018(15)$ & \\
\hline \multicolumn{7}{|l|}{ Histology } \\
\hline Not otherwise specified & $224(0)$ & $93(0)$ & $67(0)$ & $45(0)$ & $19(0)$ & \multirow[t]{5}{*}{$<.0001$} \\
\hline Large cell & $3652(5)$ & $1358(4)$ & $1225(5)$ & $695(5)$ & $374(6)$ & \\
\hline Squamous & $22,791(29)$ & $8584(25)$ & $8069(33)$ & $4390(33)$ & $1748(26)$ & \\
\hline Other & $4030(5)$ & $1604(5)$ & $1254(5)$ & $781(6)$ & $391(6)$ & \\
\hline Adenocarcinoma & $48,282(61)$ & $23,142(67)$ & $13,699(56)$ & $7227(55)$ & $4214(63)$ & \\
\hline \multicolumn{7}{|l|}{ Tumor grade } \\
\hline Well/moderately differentiated & $46,558(59)$ & $23,274(67)$ & $13,619(56)$ & $6519(50)$ & $3146(47)$ & \multirow[t]{3}{*}{$<.0001$} \\
\hline Poorly/undifferentiated & $29,030(37)$ & $9788(28)$ & $9699(40)$ & $6195(47)$ & $3348(50)$ & \\
\hline Unknown & $3391(4)$ & $1719(5)$ & $996(4)$ & $424(3)$ & $252(4)$ & \\
\hline
\end{tabular}




\begin{tabular}{|c|c|c|c|c|c|c|}
\hline Categories & $\begin{array}{c}\text { Total } \\
\mathbf{n}=\mathbf{7 8 , 9 7 9} \\
\mathbf{n}(\%) \\
\end{array}$ & $\begin{array}{c}\text { Stage IA } \\
\text { (T1ab, N0) } \\
\mathbf{n}=\mathbf{3 4 , 7 8 1} \\
\mathbf{n}(\%) \\
\end{array}$ & $\begin{array}{c}\text { Stage IB } \\
\text { (T2a, N0) and } \\
\begin{array}{c}\text { IIA (T2b, N0) } \\
\text { n }=\mathbf{2 4 , 3 1 4} \\
\text { n }(\%)\end{array}\end{array}$ & $\begin{array}{c}\text { Stage IIA } \\
\text { (T1ab-T2a, N1) } \\
\text { and IIB } \\
\mathbf{n}=\mathbf{1 3 , 1 3 8} \\
\mathbf{n}(\%)\end{array}$ & $\begin{array}{c}\text { Stage IIIA } \\
\begin{array}{c}\text { (T1-T3,N2; T3, N1) } \\
\text { n }=6746 \\
\text { n }(\%)\end{array}\end{array}$ & $\boldsymbol{P}$ \\
\hline \multicolumn{7}{|l|}{ Tumor size, $\mathrm{cm}$} \\
\hline$\leq 3$ & $49,644(63)$ & $34,452(99)$ & $6486(27)$ & $5711(44)$ & 2995 (44) & $<.0001$ \\
\hline$>3-\leq 5$ & $19,238(24)$ & $167(0)$ & $12,819(53)$ & $4188(32)$ & $2064(31)$ & \\
\hline$>5$ & $9866(13)$ & $97(0)$ & $4932(20)$ & 3175 (24) & $1662(25)$ & \\
\hline Unknown & $231(0)$ & $65(0)$ & $77(0)$ & $64(0)$ & $25(0.4)$ & \\
\hline \multicolumn{7}{|l|}{ Rural/Urban } \\
\hline Rural & $14,999(19)$ & $6312(18)$ & $4720(19)$ & $2686(20)$ & $1281(19)$ & $<.0001$ \\
\hline Urban & $59,057(75)$ & $26,236(75)$ & $18,097(74)$ & $9680(74)$ & $5044(75)$ & \\
\hline Unknown & $4923(6)$ & $2233(6)$ & $1497(6)$ & $772(6)$ & $421(6)$ & \\
\hline \multicolumn{7}{|l|}{ Census region } \\
\hline Northeast & $15,939(20)$ & $7486(22)$ & 4727 (19) & 2374 (18) & $1352(20)$ & $<.0001$ \\
\hline Midwest & $21,583(27)$ & $9160(26)$ & $6732(28)$ & 3787 (29) & $1904(28)$ & \\
\hline South & $31,840(40)$ & $13,980(40)$ & $9752(40)$ & $5417(41)$ & $2691(40)$ & \\
\hline West & $9617(12)$ & $4155(12)$ & 3103 (13) & $1560(12)$ & 799 (12) & \\
\hline \multicolumn{7}{|l|}{ Primary site } \\
\hline C340-main bronchus & $431(1)$ & $46(0)$ & $95(0)$ & $199(2)$ & $91(1)$ & $<.0001$ \\
\hline C341-upper lobe & $47,385(60)$ & $21,947(63)$ & $13,929(57)$ & 7547 (57) & $3962(59)$ & \\
\hline C342-middle lobe & $3819(5)$ & $1880(5)$ & $1119(5)$ & $562(4)$ & $258(4)$ & \\
\hline C343-lower lobe & $25,061(32)$ & $10,323(30)$ & $8340(34)$ & 4249 (32) & 2149 (32) & \\
\hline C348-overlapping lesion & $1191(2)$ & $199(1)$ & $467(2)$ & $352(3)$ & $173(3)$ & \\
\hline C349-lung not otherwise specified & $1092(1)$ & $386(1)$ & $364(2)$ & $229(2)$ & $113(2)$ & \\
\hline \multicolumn{7}{|l|}{$\mathrm{T}$ category } \\
\hline $\mathrm{T} 1$ & $40,403(51)$ & $34,781(100)$ & $0(0)$ & $3716(28)$ & $1906(28)$ & $<.0001$ \\
\hline $\mathrm{T} 2$ & $33,968(43)$ & $0(0)$ & $24,314(100)$ & $6360(48)$ & $3294(49)$ & \\
\hline $\mathrm{T} 3$ & $4608(6)$ & $0(0)$ & $0(0)$ & $3062(23)$ & $1546(23)$ & \\
\hline \multicolumn{7}{|l|}{$\mathrm{N}$ category } \\
\hline No & $62,157(79)$ & $34,781(100)$ & $24,314(100)$ & $3062(23)$ & $0(0)$ & $<.0001$ \\
\hline N1 & $11,108(14)$ & $0(0)$ & $0(0)$ & $10,076(77)$ & $1032(15)$ & \\
\hline N2 & $5714(7)$ & $0(0)$ & $0(0)$ & $0(0)$ & $5714(85)$ & \\
\hline \multicolumn{7}{|l|}{ Surgery } \\
\hline Sublobar & $7992(10)$ & $5005(14)$ & $1797(7)$ & $599(5)$ & $591(9)$ & $<.0001$ \\
\hline Lobe/bilobectomy & $67,209(85)$ & $29,544(85)$ & $21,515(89)$ & $10,907(83)$ & $5243(78)$ & \\
\hline Pneumonectomy & $3778(5)$ & $232(1)$ & $1002(4)$ & $1632(12)$ & $912(14)$ & \\
\hline \multicolumn{7}{|l|}{ Facility type } \\
\hline Community Cancer Program & $5707(7)$ & $2505(7)$ & $1746(7)$ & $1011(8)$ & $445(7)$ & .0005 \\
\hline Comprehensive Community Cancer Program & $37,952(48)$ & $16,705(48)$ & $11,724(48)$ & $6387(49)$ & $3136(47)$ & \\
\hline Teaching/Research Cancer Program & $19,304(24)$ & $8490(24)$ & $5892(24)$ & $3162(24)$ & $1760(26)$ & \\
\hline National Cancer Institute Program/Network & $8954(11)$ & $4052(12)$ & $2738(11)$ & $1395(11)$ & $769(11)$ & \\
\hline Other & $7062(9)$ & $3029(9)$ & $2214(9)$ & $1183(9)$ & $636(9)$ & \\
\hline
\end{tabular}


TABLE E3. Patient demographic and institutional characteristics among margin-negative patients by adjuvant therapy

\begin{tabular}{|c|c|c|c|c|c|c|}
\hline Categories & $\begin{array}{c}\text { Total } \\
\mathbf{n}=\mathbf{7 8 , 9 7 9} \\
\mathbf{n}(\%) \\
\end{array}$ & $\begin{array}{c}\text { No treatment } \\
\mathbf{n}=\mathbf{6 1 , 2 8 1} \\
\mathbf{n}(\%)\end{array}$ & $\begin{array}{c}\text { Chemotherapy } \\
\begin{array}{c}\mathbf{n}=\mathbf{1 3 , 6 6 5} \\
\text { n }(\%)\end{array}\end{array}$ & $\begin{array}{c}\text { Radiation therapy } \\
\mathbf{n}=\mathbf{9 8 8} \\
\mathbf{n}(\%)\end{array}$ & $\begin{array}{c}\text { Chemoradiation } \\
\text { n }=\mathbf{3 0 4 5} \\
\mathbf{n}(\%)\end{array}$ & $\boldsymbol{P}$ \\
\hline \multicolumn{7}{|l|}{ Stage group } \\
\hline Stage IA & $34,781(44)$ & $33,780(55)$ & $789(6)$ & $136(14)$ & $76(3)$ & \multirow[t]{4}{*}{$<.0001$} \\
\hline Stage IB and IIA & $24,314(31)$ & $19,281(32)$ & $4568(33)$ & $250(25)$ & $215(7)$ & \\
\hline Stage IIA and IIB & $13,138(17)$ & $6101(10)$ & $5788(42)$ & $354(36)$ & $895(29)$ & \\
\hline Stage IIIA & $6746(9)$ & $2119(4)$ & $2520(18)$ & $248(25)$ & $1859(61)$ & \\
\hline \multicolumn{7}{|l|}{ Age group } \\
\hline $18-49$ & $4389(6)$ & $2882(5)$ & $1114(8)$ & $56(6)$ & $337(11)$ & \multirow[t]{4}{*}{$<.0001$} \\
\hline $50-64$ & $25,864(33)$ & $18,296(30)$ & $5962(44)$ & $261(26)$ & $1345(44)$ & \\
\hline $65-74$ & $29,782(38)$ & $23,397(38)$ & $4981(37)$ & $382(39)$ & $1022(34)$ & \\
\hline $75-90$ & $18,944(24)$ & $16,706(27)$ & $1608(12)$ & $289(29)$ & $341(11)$ & \\
\hline \multicolumn{7}{|l|}{ Sex } \\
\hline Male & $37,844(48)$ & $28,854(47)$ & $6856(50)$ & $547(55)$ & $1587(52)$ & \multirow[t]{2}{*}{$<.0001$} \\
\hline Female & $41,135(52)$ & $32,427(53)$ & $6809(50)$ & $441(45)$ & $1458(48)$ & \\
\hline \multicolumn{7}{|l|}{ Race/Ethnicity } \\
\hline Non-Hispanic, white & $61,957(79)$ & $48,361(79)$ & $10,515(77)$ & $758(77)$ & $2323(76)$ & \multirow[t]{5}{*}{$<.0001$} \\
\hline Hispanic & $1734(2)$ & $1356(2)$ & $297(2)$ & $17(2)$ & $64(2)$ & \\
\hline Black & $6478(8)$ & $4803(8)$ & $1293(10)$ & $96(10)$ & $286(9)$ & \\
\hline Other & $2078(3)$ & $1616(3)$ & $346(3)$ & $28(3)$ & $88(3)$ & \\
\hline Missing & $6732(9)$ & $5145(8)$ & $1214(9)$ & $89(9)$ & $284(9)$ & \\
\hline \multicolumn{7}{|l|}{ Insurance } \\
\hline Uninsured & $1517(2)$ & $1091(2)$ & $341(3)$ & $16(2)$ & $69(2)$ & \multirow[t]{6}{*}{$<.0001$} \\
\hline Medicaid & $3325(4)$ & $2382(4)$ & $720(5)$ & $51(5)$ & $172(6)$ & \\
\hline Younger Medicare & $4538(6)$ & $3413(6)$ & $849(6)$ & $59(6)$ & $217(7)$ & \\
\hline Older Medicare & $41,134(52)$ & $33,907(55)$ & $5528(41)$ & $576(58)$ & $1123(37)$ & \\
\hline Private & $27,402(35)$ & $19,609(32)$ & $6083(45)$ & $277(28)$ & $1433(47)$ & \\
\hline Missing & $1063(1)$ & $879(1)$ & $144(1)$ & $9(1)$ & $31(1)$ & \\
\hline \multicolumn{7}{|l|}{ Median income-quartile } \\
\hline$<\$ 30,000$ & $10,308(13)$ & $7968(13)$ & $1808(13)$ & $158(16)$ & $374(12)$ & \multirow[t]{5}{*}{$<.0001$} \\
\hline$\$ 30,000-\$ 34,999$ & $14,718(19)$ & $11,386(19)$ & $2498(18)$ & $221(22)$ & $613(20)$ & \\
\hline$\$ 35,000-\$ 45,999$ & $21,718(28)$ & $16,705(27)$ & $3878(28)$ & $270(27)$ & $865(28)$ & \\
\hline$\$ 46,000+$ & $27,950(35)$ & $21,822(36)$ & $4807(35)$ & $300(30)$ & $1021(34)$ & \\
\hline Missing & $4285(5)$ & $3400(6)$ & $674(5)$ & $39(4)$ & $172(6)$ & \\
\hline \multicolumn{7}{|l|}{ Comorbidity } \\
\hline 0 & $37,470(47)$ & $28,612(47)$ & $6848(50)$ & $438(44)$ & $1572(52)$ & \multirow[t]{3}{*}{$<.0001$} \\
\hline 1 & $28,532(36)$ & $22,149(36)$ & $4945(36)$ & $364(37)$ & $1074(35)$ & \\
\hline $2+$ & $12,977(16)$ & $10,520(17)$ & $1872(14)$ & $186(19)$ & $399(13)$ & \\
\hline \multicolumn{7}{|l|}{ Histology } \\
\hline Not otherwise specified & $224(0)$ & $175(0)$ & $36(0)$ & $2(0)$ & $11(0)$ & \multirow[t]{5}{*}{$<.0001$} \\
\hline Large cell & $3652(5)$ & $2581(4)$ & $821(6)$ & $64(7)$ & $186(6)$ & \\
\hline Squamous & $22,791(29)$ & $17,710(29)$ & $3897(29)$ & $383(39)$ & $801(26)$ & \\
\hline Other & $4030(5)$ & $3031(5)$ & $739(5)$ & $62(6)$ & $198(7)$ & \\
\hline Adenocarcinoma & $48,282(61)$ & $37,784(62)$ & $8172(60)$ & $477(48)$ & $1849(61)$ & \\
\hline \multicolumn{7}{|l|}{ Tumor grade } \\
\hline Well/moderately differentiated & $46,558(59)$ & $37,923(62)$ & $6827(50)$ & $496(50)$ & $1312(43)$ & \multirow[t]{3}{*}{$<.0001$} \\
\hline Poorly/undifferentiated & $29,030(37)$ & $20,626(34)$ & $6356(47)$ & 449 (46) & $1599(53)$ & \\
\hline Unknown & $3391(4)$ & $2732(5)$ & $482(4)$ & $43(4)$ & $134(4)$ & \\
\hline \multicolumn{7}{|l|}{ Tumor size, $\mathrm{cm}$} \\
\hline$\leq 3$ & $49,644(63)$ & $42,437(69)$ & $5367(39)$ & $448(45)$ & $1392(46)$ & \multirow[t]{4}{*}{$<.0001$} \\
\hline$>3-\leq 5$ & $19,238(24)$ & $13,070(21)$ & $4923(36)$ & $324(33)$ & $921(30)$ & \\
\hline$>5$ & $9866(13)$ & $5600(9)$ & $3335(24)$ & $210(21)$ & $721(24)$ & \\
\hline Unknown & $231(0)$ & $174(0)$ & $40(0)$ & $6(1)$ & $11(0)$ & \\
\hline
\end{tabular}


TABLE E3. Continued

\begin{tabular}{|c|c|c|c|c|c|c|}
\hline Categories & $\begin{array}{c}\text { Total } \\
\mathbf{n}=\mathbf{7 8 , 9 7 9} \\
\mathbf{n}(\%) \\
\end{array}$ & $\begin{array}{c}\text { No treatment } \\
\mathrm{n}=61,281 \\
\mathrm{n}(\%)\end{array}$ & $\begin{array}{c}\text { Chemotherapy } \\
\begin{array}{c}\mathbf{n}=13,665 \\
\text { n }(\%)\end{array}\end{array}$ & $\begin{array}{c}\text { Radiation therapy } \\
\mathbf{n}=\mathbf{9 8 8} \\
\mathbf{n}(\%)\end{array}$ & $\begin{array}{c}\text { Chemoradiation } \\
\begin{array}{c}\mathbf{n}=\mathbf{3 0 4 5} \\
\mathbf{n}(\%)\end{array}\end{array}$ & $P$ \\
\hline \multicolumn{7}{|l|}{ Rural/Urban } \\
\hline Rural & $14,999(19)$ & $11,672(19)$ & 2540 (19) & $196(20)$ & $591(19)$ & \multirow[t]{3}{*}{.034} \\
\hline Urban & $59,057(75)$ & $45,716(75)$ & $10,335(76)$ & $746(76)$ & $2260(74)$ & \\
\hline Unknown & $4923(6)$ & $3893(6)$ & $790(6)$ & $46(5)$ & $194(6)$ & \\
\hline \multicolumn{7}{|l|}{ Census region } \\
\hline Northeast & $15,939(20)$ & $12,446(20)$ & $2734(20)$ & $161(16)$ & $598(20)$ & \multirow[t]{4}{*}{$<.0001$} \\
\hline Midwest & $21,583(27)$ & $15,988(26)$ & $4355(32)$ & $268(27)$ & $972(32)$ & \\
\hline South & $31,840(40)$ & $24,965(41)$ & $5215(38)$ & $432(44)$ & $1228(40)$ & \\
\hline West & $9617(12)$ & $7882(13)$ & $1361(10)$ & $127(13)$ & $247(8)$ & \\
\hline \multicolumn{7}{|l|}{ Primary site } \\
\hline C340-main bronchus & $431(1)$ & $250(0)$ & $152(1)$ & $6(1)$ & $23(1)$ & \multirow[t]{6}{*}{$<.0001$} \\
\hline C341-upper lobe & $47,385(60)$ & $36,984(60)$ & $7838(57)$ & $626(63)$ & $1937(64)$ & \\
\hline C342-middle lobe & $3819(5)$ & $3024(5)$ & $617(5)$ & $40(4)$ & $138(5)$ & \\
\hline C343-lower lobe & $25,061(32)$ & $19,451(32)$ & $4509(33)$ & $278(28)$ & $823(27)$ & \\
\hline C348-overlapping lesion & $1191(2)$ & $768(1)$ & $333(2)$ & $19(2)$ & $71(2)$ & \\
\hline C349-lung not otherwise specified & $1092(1)$ & $804(1)$ & $216(2)$ & $19(2)$ & $53(2)$ & \\
\hline \multicolumn{7}{|l|}{$\mathrm{T}$ category } \\
\hline $\mathrm{T} 1$ & $40,403(51)$ & $35,961(59)$ & $3285(24)$ & $261(26)$ & $896(29)$ & \multirow[t]{3}{*}{$<.0001$} \\
\hline $\mathrm{T} 2$ & $33,968(43)$ & $23,130(38)$ & $8800(64)$ & $494(50)$ & $1544(51)$ & \\
\hline $\mathrm{T} 3$ & $4608(6)$ & $2190(4)$ & $1580(12)$ & $233(24)$ & $605(20)$ & \\
\hline \multicolumn{7}{|l|}{ N Category } \\
\hline No & $62,157(79)$ & $54,703(89)$ & $6292(46)$ & $565(57)$ & $597(20)$ & \multirow[t]{3}{*}{$<.0001$} \\
\hline N1 & $11,108(14)$ & $4855(8)$ & $5317(39)$ & $210(21)$ & $726(24)$ & \\
\hline N2 & $5714(7)$ & $1723(3)$ & $2056(15)$ & $213(22)$ & $1722(57)$ & \\
\hline \multicolumn{7}{|l|}{ Surgery } \\
\hline Sublobar & $7992(10)$ & $6738(11)$ & $718(5)$ & $169(17)$ & $367(12)$ & \multirow[t]{3}{*}{$<.0001$} \\
\hline Lobe/bilobectomy & $67,209(85)$ & $52,526(86)$ & $11,513(84)$ & $744(75)$ & $2426(80)$ & \\
\hline Pneumonectomy & $3778(5)$ & $2017(3)$ & $1434(11)$ & $75(8)$ & $252(8)$ & \\
\hline \multicolumn{7}{|l|}{ Facility type } \\
\hline Community Cancer Program & $5707(7)$ & $4283(7)$ & $1023(8)$ & $112(11)$ & $289(10)$ & \multirow[t]{5}{*}{$<.0001$} \\
\hline Comprehensive Community Cancer Program & $37,952(48)$ & $29,420(48)$ & $6456(47)$ & $500(51)$ & $1576(52)$ & \\
\hline Teaching/Research Cancer Program & $19,304(24)$ & $15,141(25)$ & $3235(24)$ & $214(22)$ & $714(24)$ & \\
\hline National Cancer Institute Program/Network & $8954(11)$ & $7039(12)$ & $1657(12)$ & $67(7)$ & $191(6)$ & \\
\hline Other & $7062(9)$ & $5398(9)$ & $1294(10)$ & $95(10)$ & $275(9)$ & \\
\hline
\end{tabular}


TABLE E4. Numbers of events in each analysis group

\begin{tabular}{|c|c|c|c|c|c|}
\hline & \multirow[b]{2}{*}{ Postoperative treatment } & \multicolumn{2}{|c|}{ Margin positive } & \multicolumn{2}{|c|}{ Margin negative } \\
\hline & & $\mathbf{n}$ & Event $=$ death & $\mathbf{n}$ & Event $=$ death \\
\hline \multirow[t]{4}{*}{ Group 1: stage IA (T1ab, N0) } & No treatment & 265 & $75(28.3)$ & 33,780 & $5695(16.86)$ \\
\hline & Chemo only & 19 & $6(31.58)$ & 789 & $157(19.9)$ \\
\hline & Radiation only & 60 & $25(41.67)$ & 136 & $55(40.44)$ \\
\hline & Chemo + Rad & 25 & $12(48)$ & 76 & $29(28.16)$ \\
\hline \multirow{4}{*}{$\begin{array}{c}\text { Group 2: stage IB (T2a, N0) } \\
\text { and stage IIA (T2b, N0) }\end{array}$} & No treatment & 477 & $182(38.16)$ & 19,281 & $5427(28.15)$ \\
\hline & Chemo only & 142 & $39(27.46)$ & 4568 & $996(21.8)$ \\
\hline & Radiation only & 119 & $62(52.1)$ & 250 & $114(45.6)$ \\
\hline & Chemo + Rad & 119 & $53(44.54)$ & 215 & $88(40.93)$ \\
\hline \multirow{7}{*}{$\begin{array}{l}\text { Group 3: stage IIA (T1ab- } \\
\text { T2a, N1) and stage IIB (T3, } \\
\text { N0; T2b, N1) }\end{array}$} & No treatment & 419 & $239(57.04)$ & 6101 & $2663(43.65)$ \\
\hline & Chemo only & 284 & $129(45.42)$ & 5788 & $1691(29.22)$ \\
\hline & Radiation only & 199 & $121(60.8)$ & 354 & $198(55.93)$ \\
\hline & Chemo + Rad & 415 & $186(44.82)$ & 895 & $398(44.47)$ \\
\hline & Chemo + Rad & 146 & $69(47.26)$ & 431 & $186(43.16)$ \\
\hline & $\operatorname{Rad}+$ Chemo & 120 & $56(46.67)$ & 224 & 109 (48.66) \\
\hline & Chemo $=\operatorname{Rad}$ & 149 & $61(40.94)$ & 240 & $103(42.92)$ \\
\hline \multirow{7}{*}{$\begin{array}{l}\text { Group 4: stage IIIA (T1-3, } \\
\text { N2; T3, N1) }\end{array}$} & No treatment & 245 & $170(69.39)$ & 2119 & $1218(57.48)$ \\
\hline & Chemo only & 200 & $107(53.5)$ & 2520 & $969(38.45)$ \\
\hline & Radiation only & 69 & $53(76.81)$ & 248 & $151(60.89)$ \\
\hline & Chemo + Rad & 404 & $213(52.72)$ & 1859 & $791(42.55)$ \\
\hline & Chemo + Rad & 173 & $90(52.02)$ & 1096 & $426(38.87)$ \\
\hline & $\operatorname{Rad}+$ Chemo & 96 & $54(56.25)$ & 316 & $150(47.47)$ \\
\hline & Chemo $=\operatorname{Rad}$ & 135 & $69(51.11)$ & 447 & $215(48.1)$ \\
\hline
\end{tabular}

Chemo, Chemotherapy; Rad, radiation.

TABLE E5. Propensity score-adjusted models

\begin{tabular}{|c|c|c|c|c|c|c|c|}
\hline & \multirow[b]{2}{*}{$\begin{array}{l}\text { Postoperative } \\
\text { treatment }\end{array}$} & \multicolumn{3}{|c|}{ Margin positive } & \multicolumn{3}{|c|}{ Margin negative } \\
\hline & & $\mathbf{n}$ & $\begin{array}{c}\text { Propensity-adjusted } \\
\text { hazard ratio }(95 \% \mathrm{CI})\end{array}$ & $\boldsymbol{P}$ & n & $\begin{array}{c}\text { Propensity-adjusted } \\
\text { hazard ratio }(95 \% \mathrm{CI})\end{array}$ & $\boldsymbol{P}$ \\
\hline \multirow[t]{4}{*}{ Group 1: stage IA (T1ab, N0) } & No treatment & 265 & 1.00 (Referent) & & 33,780 & 1.00 (Referent) & \\
\hline & Chemo only & 19 & $0.94(0.36-2.44)$ & .8968 & 789 & $1.02(0.87-1.20)$ & .7841 \\
\hline & Radiation only & 60 & $1.87(1.16-3.04)$ & .0110 & 136 & $2.37(1.81-3.09)$ & $<.0001$ \\
\hline & Chemo + Rad & 25 & $1.39(0.73-2.63)$ & .3142 & 76 & $2.91(2.02-4.19)$ & $<.0001$ \\
\hline \multirow{4}{*}{$\begin{array}{l}\text { Group 2: stage IB (T2a, N0) } \\
\text { and } \\
\text { stage IIA (T2b, N0) }\end{array}$} & No treatment & 477 & 1.00 (Referent) & & 19,281 & 1.00 (Referent) & \\
\hline & Chemo only & 142 & $0.58(0.40-0.83)$ & .0032 & 4568 & $0.73(0.68-0.78)$ & $<.0001$ \\
\hline & Radiation only & 119 & $1.30(0.97-1.73)$ & .0818 & 250 & $1.79(1.49-2.16)$ & $<.0001$ \\
\hline & Chemo + Rad & 119 & $1.01(0.73-1.39)$ & .9688 & 215 & $1.42(1.15-1.76)$ & .0012 \\
\hline \multirow{7}{*}{$\begin{array}{l}\text { Group 3: stage IIA (T1ab- } \\
\text { T2a, N1) and } \\
\text { stage IIB (T3, N0; T2b, N1) }\end{array}$} & No treatment & 419 & 1.00 (Referent) & & 6101 & 1.00 (Referent) & \\
\hline & Chemo only & 284 & $0.73(0.59-0.91)$ & .0058 & 5788 & $0.66(0.62-0.71)$ & $<.0001$ \\
\hline & Radiation only & 199 & $1.02(0.81-1.28)$ & .8649 & 354 & $1.37(1.19-1.59)$ & $<.0001$ \\
\hline & Chemo + Rad & 415 & $0.78(0.64-0.97)$ & .0218 & 895 & $1.05(0.95-1.18)$ & .3410 \\
\hline & Chemo + Rad & 146 & $0.81(0.61-1.07)$ & .1343 & 431 & $1.04(0.89-1.20)$ & .6584 \\
\hline & Rad + Chemo & 120 & 0.77 (0.57-1.06) & .1054 & 224 & $1.12(0.93-1.37)$ & .2400 \\
\hline & Chemo $=\operatorname{Rad}$ & 149 & $0.80(0.59-1.07)$ & .1344 & 240 & $1.06(0.86-1.29)$ & .5967 \\
\hline \multirow{7}{*}{$\begin{array}{l}\text { Group 4: stage IIIA (T1-3, } \\
\text { N2; T3, N1) }\end{array}$} & No treatment & 245 & 1.00 (Referent) & & 2119 & 1.00 (Referent) & \\
\hline & Chemo only & 200 & $0.74(0.58-0.95)$ & .0167 & 2520 & $0.64(0.58-0.69)$ & $<.0001$ \\
\hline & Radiation only & 69 & $1.13(0.83-1.54)$ & .4428 & 248 & $1.17(0.98-1.38)$ & .0757 \\
\hline & Chemo + Rad & 404 & $0.67(0.54-0.83)$ & .0003 & 1859 & $0.71(0.65-0.79)$ & $<.0001$ \\
\hline & Chemo + Rad & 173 & $0.59(0.45-0.77)$ & .0001 & 1096 & $0.64(0.56-0.71)$ & $<.0001$ \\
\hline & $\operatorname{Rad}+$ Chemo & 96 & $0.79(0.57-1.09)$ & .1434 & 316 & $0.77(0.65-0.92)$ & .0038 \\
\hline & Chemo $=\mathrm{Rad}$ & 135 & $0.59(0.44-0.80)$ & .0006 & 447 & $0.78(0.67-0.90)$ & .0010 \\
\hline
\end{tabular}

CI, Confidence interval; Chemo, chemotherapy; Rad, radiation. 
TABLE E6. Proportional hazards models by stage group and margin status after removal of exposure groups where the proportional hazards assumption is questionable

\begin{tabular}{|c|c|c|c|c|c|}
\hline & \multirow[b]{2}{*}{ Postoperative treatment } & \multicolumn{4}{|c|}{ Margin positive } \\
\hline & & $\mathbf{n}$ & $\begin{array}{l}\text { Unadjusted hazard } \\
\text { ratio }(95 \% \mathbf{C I})\end{array}$ & $\begin{array}{c}\text { Adjusted } \\
\text { hazard ratio }(95 \% \mathrm{CI})\end{array}$ & $\boldsymbol{P}$ \\
\hline \multirow[t]{4}{*}{ Group 1: stage IA (T1ab, N0) } & No treatment & 265 & 1.00 (Referent) & 1.00 (Referent) & \\
\hline & \multicolumn{5}{|c|}{ Chemo only (patients who had chemotherapy only were excluded from the analysis) } \\
\hline & Radiation only & 60 & $1.98(1.25-3.12)$ & $1.75(1.03-2.97)$ & .0385 \\
\hline & Chemo + Rad & 25 & $1.69(0.92-3.11)$ & $0.92(0.44-1.92)$ & .8202 \\
\hline \multirow{4}{*}{$\begin{array}{l}\text { Group 3: stage IIA (T1ab-T2a, N1) } \\
\text { and stage IIB (T3, N0; T2b, N1) }\end{array}$} & No treatment & 419 & 1.00 (Referent) & 1.00 (Referent) & \\
\hline & Chemo only & 284 & $0.65(0.53-0.81)$ & $0.72(0.58-0.90)$ & .0042 \\
\hline & \multicolumn{5}{|c|}{ Radiation only (patients who had radiation therapy only were excluded from the analysis) } \\
\hline & Chemo + Rad & 415 & $0.68(0.56-0.83)$ & $0.77(0.63-0.95)$ & .0148 \\
\hline
\end{tabular}

Bold indicates $P<.05$. CI, Confidence interval; Chemo, chemotherapy; Rad, radiation.

TABLE E7. Analysis including only anatomic resections

\begin{tabular}{|c|c|c|c|c|}
\hline & \multirow[b]{2}{*}{ Postoperative treatment } & \multicolumn{3}{|c|}{ Margin positive } \\
\hline & & $\mathbf{n}$ & Adjusted hazard ratio $(95 \%$ CI) & $P$ \\
\hline \multirow[t]{4}{*}{ Group 1: stage IA (T1ab, N0) } & No treatment & 197 & 1.00 (Referent) & \\
\hline & Chemo only & 13 & $0.86(0.18-4.16)$ & .8501 \\
\hline & Radiation only & 31 & $4.14(1.88-9.09)$ & .0004 \\
\hline & Chemo + Rad & 14 & $1.14(0.38-3.44)$ & .8125 \\
\hline \multirow{4}{*}{$\begin{array}{l}\text { Group 2: stage IB (T2a, N0) } \\
\text { and stage IIA (T2b, N0) }\end{array}$} & No treatment & 423 & 1.00 (Referent) & \\
\hline & Chemo only & 131 & $0.54(0.37-0.80)$ & .0023 \\
\hline & Radiation only & 100 & $1.33(0.96-1.85)$ & .0899 \\
\hline & Chemo + Rad & 108 & $0.94(0.66-1.32)$ & .7036 \\
\hline \multirow{4}{*}{$\begin{array}{l}\text { Group 3: stage IIA (T1ab- } \\
\text { T2a, N1) and stage IIB (T3, } \\
\text { N0;T2b, N1) }\end{array}$} & No treatment & 386 & 1.00 (Referent) & \\
\hline & Chemo only & 266 & $0.75(0.60-0.95)$ & .016 \\
\hline & Radiation only & 177 & $0.99(0.77-1.27)$ & .9379 \\
\hline & Chemo + Rad & 379 & $0.80(0.64-0.99)$ & .0389 \\
\hline \multirow{4}{*}{$\begin{array}{l}\text { Group 4: stage IIIA (T1-3, } \\
\text { N2; T3, N1) }\end{array}$} & No treatment & 220 & 1.00 (Referent) & \\
\hline & Chemo only & 179 & $0.73(0.56-0.96)$ & .0229 \\
\hline & Radiation only & 61 & $1.05(0.74-1.48)$ & .7959 \\
\hline & Chemo + Rad & 356 & $0.66(0.53-0.84)$ & .0006 \\
\hline
\end{tabular}

CI, Confidence interval; Chemo, chemotherapy; Rad, radiation. 
TABLE E8. Kaplan-Meier survival analysis and proportional hazards models by stage group for margin-positive patients evaluating the order of chemotherapy and radiation

\begin{tabular}{|c|c|c|c|c|c|c|}
\hline & \multirow[b]{2}{*}{$\begin{array}{c}\text { Postoperative } \\
\text { treatment }\end{array}$} & \multicolumn{5}{|c|}{ Margin positive } \\
\hline & & $\mathbf{n}$ & $\begin{array}{c}\text { 5-y overall } \\
\text { survival (log-rank } \\
P \text { value) } \\
\end{array}$ & $\begin{array}{c}\text { Unadjusted hazard } \\
\text { ratio }(95 \% \mathrm{CI}) \\
\end{array}$ & $\begin{array}{c}\text { Adjusted hazard } \\
\text { ratio }(95 \% \mathrm{CI}) \\
\end{array}$ & $P$ \\
\hline \multirow{7}{*}{$\begin{array}{l}\text { Group 3: stage IIA } \\
\text { (T1ab-T2a, N1) and stage } \\
\text { IIB (T3, N0; T2b, N1) }\end{array}$} & No treatment & 419 & 27 (Referent) & 1.00 (Referent) & 1.00 (Referent) & \\
\hline & Chemo only & 284 & $36(.0001)$ & $0.65(0.53-0.81)$ & $0.72(0.58-0.9)$ & .0041 \\
\hline & Radiation only & 199 & $26(.5907)$ & $1.06(0.85-1.32)$ & $0.94(0.74-1.18)$ & .5878 \\
\hline & Chemo + Rad & 415 & $37(<.0001)$ & $0.68(0.56-0.83)$ & $0.76(0.62-0.93)$ & .0083 \\
\hline & Chemo + Rad & 146 & $37(.0106)$ & $0.70(0.54-0.92)$ & $0.74(0.56-0.98)$ & .0340 \\
\hline & $\operatorname{Rad}+$ Chemo & 120 & $36(.0136)$ & $0.69(0.52-0.92)$ & $0.76(0.56-1.02)$ & .0710 \\
\hline & Chemo $=\operatorname{Rad}$ & 149 & $38(.0036)$ & $0.66(0.50-0.87)$ & $0.79(0.59-1.06)$ & .1099 \\
\hline \multirow{7}{*}{$\begin{array}{l}\text { Group 4: stage IIIA (T1-3, } \\
\text { N2; T3, N1) }\end{array}$} & No treatment & 245 & 12 (Referent) & 1.00 (Referent) & 1.00 (Referent) & \\
\hline & Chemo only & 200 & $21(.0048)$ & $0.70(0.55-0.90)$ & $0.77(0.6-1)$ & .0466 \\
\hline & Radiation only & 69 & $10(.5215)$ & $1.11(0.81-1.51)$ & $1.03(0.74-1.43)$ & .8729 \\
\hline & Chemo + Rad & 404 & $25(<.0001)$ & $0.59(0.48-0.72)$ & $0.63(0.51-0.79)$ & $<.0001$ \\
\hline & Chemo + Rad & 173 & $28(<.0001)$ & $0.54(0.42-0.70)$ & $0.56(0.43-0.74)$ & $<.0001$ \\
\hline & $\operatorname{Rad}+$ Chemo & 96 & $19(.0275)$ & $0.70(0.52-0.95)$ & $0.79(0.57-1.09)$ & .1508 \\
\hline & Chemo $=\mathrm{Rad}$ & 135 & $24(.0002)$ & $0.58(0.44-0.77)$ & $0.63(0.46-0.84)$ & .0021 \\
\hline
\end{tabular}

CI, Confidence interval; Chemo, chemotherapy; Rad, radiation.

TABLE E9. Survival results for margin-positive patients by R1, R2, and R-unknown

\begin{tabular}{|c|c|c|c|c|c|c|c|c|c|}
\hline \multirow[b]{3}{*}{$\begin{array}{c}\text { Margin } \\
\text { status } \\
\end{array}$} & \multirow[b]{3}{*}{$\begin{array}{c}\text { Postoperative } \\
\text { treatment }\end{array}$} & \multicolumn{4}{|c|}{ Stage IIA (T1ab-T2a, N1) and stage IIB (T3, N0;T2b, N1) } & \multicolumn{4}{|c|}{ Stage IIIA (T1-3, N2; T3, N1) } \\
\hline & & \multicolumn{4}{|c|}{ Margin positive $(n=1327)$} & \multicolumn{4}{|c|}{ Margin positive $(n=919)$} \\
\hline & & Total n & $\begin{array}{l}\text { 5-y overall survival } \\
\text { (log-rank } P \text { value)* }\end{array}$ & $\begin{array}{c}\text { Adjusted hazard } \\
\text { ratio }(95 \% \mathrm{CI})\end{array}$ & $P$ value & Total $\mathbf{n}$ & $\begin{array}{c}\text { 5-y overall } \\
\text { survival } \\
(\log -\text {-rank } P \text { value })^{*}\end{array}$ & $\begin{array}{c}\text { Adjusted hazard } \\
\text { ratio }(95 \% \mathbf{C I})\end{array}$ & $P$ value \\
\hline \multirow[t]{4}{*}{$\mathrm{R} 1$} & No treatment & 231 & 26 (referent) & 1.00 (Referent) & & 127 & 15 (referent) & 1.00 (Referent) & \\
\hline & Chemo only & 146 & $36(.0024)$ & $0.65(0.47-0.88)$ & .0063 & 121 & $19(0.1362)$ & $0.83(0.59-1.17)$ & .2876 \\
\hline & Radiation only & 135 & $29(.5925)$ & $0.83(0.61-1.13)$ & .2376 & 29 & $13(0.9215)$ & $0.97(0.57-1.62)$ & .8925 \\
\hline & Chemo + Rad & 241 & $37(.0019)$ & $0.66(0.5-0.87)$ & .0036 & 237 & $23(0.001)$ & $0.66(0.48-0.91)$ & .0101 \\
\hline \multirow[t]{4}{*}{ R2 } & No treatment & 13 & 23 (referent) & ID $*$ & & 14 & 32 (referent) & $\mathrm{ID}^{*}$ & \\
\hline & Chemo only & 12 & $48(.0833)$ & ID & & 5 & $40(.9666)$ & ID & \\
\hline & Radiation only & 6 & 0 (.6098) & ID & & 6 & $17(.5783)$ & ID & \\
\hline & Chemo $+\operatorname{Rad}$ & 22 & $35(.1247)$ & ID & & 18 & $9(.6722)$ & ID & \\
\hline \multirow[t]{4}{*}{$\mathrm{RX}$} & No treatment & 178 & 31 (referent) & 1.00 (Referent) & & 105 & 8 (referent) & 1.00 (Referent) & \\
\hline & Chemo only & 129 & $35(.0503)$ & $0.78(0.55-1.11)$ & .1703 & 78 & $25(.0075)$ & $0.60(0.38-0.95)$ & .0284 \\
\hline & Radiation only & 61 & $23(.1165)$ & $1.16(0.76-1.76)$ & .4983 & 35 & $8(.7908)$ & $1.13(0.67-1.91)$ & .6458 \\
\hline & Chemo $+\operatorname{Rad}$ & 153 & $37(.0649)$ & $1.10(0.77-1.57)$ & .6078 & 144 & $30(<.0001)$ & $0.48(0.33-0.69)$ & $<.0001$ \\
\hline
\end{tabular}

$C I$, Confidence interval; Chemo, chemotherapy; Rad, radiation; $I D$, insufficient data. ${ }^{*} P$ value from the log-rank test, comparing the entire survival curves. 
TABLE E10. Kaplan-Meier survival analysis and proportional hazards models by stage group for margin-negative patients evaluating the order of chemotherapy and radiation

\begin{tabular}{|c|c|c|c|c|c|c|}
\hline & \multirow[b]{2}{*}{$\begin{array}{c}\text { Postoperative } \\
\text { treatment }\end{array}$} & \multicolumn{5}{|c|}{ Margin negative } \\
\hline & & $\mathbf{n}$ & $\begin{array}{c}\text { 5-y overall survival } \\
\text { (log-rank } P \text { value) }\end{array}$ & $\begin{array}{l}\text { Unadjusted hazard } \\
\text { ratio }(95 \% \mathrm{CI})\end{array}$ & $\begin{array}{c}\text { Adjusted hazard } \\
\text { ratio }(95 \% \mathrm{CI})\end{array}$ & $\boldsymbol{P}$ \\
\hline \multirow[t]{4}{*}{ Group 1: stage IA (T1ab, N0) } & No treatment & 33,780 & 72 (Referent) & 1.00 (Referent) & 1.00 (Referent) & \\
\hline & Chemo only & 789 & $74(.2946)$ & $0.92(0.78-1.08)$ & $1.02(0.87-1.2)$ & .8160 \\
\hline & Radiation only & 136 & $44(<.0001)$ & $2.89(2.22-3.73)$ & $2.18(1.67-2.85)$ & $<.0001$ \\
\hline & Chemo + Rad & 76 & $40(<.0001)$ & $3.19(2.21-4.59)$ & $2.99(2.07-4.31)$ & $<.0001$ \\
\hline \multirow{4}{*}{$\begin{array}{l}\text { Group 2: stage IB (T2a, N0) and } \\
\text { stage IIA (T2b, N0) }\end{array}$} & No treatment & 19,281 & 57 (Referent) & 1.00 (Referent) & 1.00 (Referent) & \\
\hline & Chemo only & 4568 & $68(<.0001)$ & $0.68(0.63-0.72)$ & $0.74(0.69-0.8)$ & $<.0001$ \\
\hline & Radiation only & 250 & $38(<.0001)$ & $1.92(1.60-2.31)$ & $1.8(1.49-2.16)$ & $<.0001$ \\
\hline & Chemo + Rad & 215 & $47(.0003)$ & $1.47(1.19-1.81)$ & $1.41(1.14-1.74)$ & .0016 \\
\hline \multirow{7}{*}{$\begin{array}{l}\text { Group 3: stage IIA (T1ab-T2a, N1) and } \\
\text { stage IIB (T3, N0;T2b, N1) }\end{array}$} & No treatment & 6101 & 37 (Referent) & 1.00 (Referent) & 1.00 (Referent) & \\
\hline & Chemo only & 5788 & $53(<.0001)$ & $0.59(0.56-0.63)$ & $0.66(0.62-0.7)$ & $<.0001$ \\
\hline & Radiation only & 354 & $28(<.0001)$ & $1.35(1.17-1.57)$ & $1.36(1.18-1.58)$ & $<.0001$ \\
\hline & Chemo + Rad & 895 & $41(.1772)$ & $0.93(0.84-1.03)$ & $1.04(0.93-1.16)$ & .4811 \\
\hline & Chemo + Rad & 431 & $42(.1966)$ & $0.91(0.78-1.05)$ & $1(0.86-1.16)$ & .9780 \\
\hline & Rad + Chemo & 224 & $36(.9445)$ & $0.99(0.82-1.20)$ & $1.13(0.93-1.37)$ & .2090 \\
\hline & Chemo $=\operatorname{Rad}$ & 240 & $42(.3621)$ & $0.91(0.75-1.11)$ & $1.03(0.84-1.26)$ & .7820 \\
\hline \multirow[t]{7}{*}{ Group 4: stage IIIA (T1-3, N2; T3, N1) } & No treatment & 2119 & 24 (Referent) & 1.00 (Referent) & 1.00 (Referent) & \\
\hline & Chemo only & 2520 & $39(<.0001)$ & $0.59(0.55-0.65)$ & $0.63(0.58-0.69)$ & $<.0001$ \\
\hline & Radiation only & 248 & $18(.0747)$ & $1.18(0.99-1.39)$ & $1.15(0.97-1.37)$ & .1025 \\
\hline & Chemo + Rad & 1859 & $39(<.0001)$ & $0.62(0.57-0.68)$ & $0.69(0.63-0.76)$ & $<.0001$ \\
\hline & Chemo + Rad & 1096 & $42(<.0001)$ & $0.56(0.50-0.62)$ & $0.62(0.56-0.7)$ & $<.0001$ \\
\hline & $\operatorname{Rad}+$ Chemo & 316 & $34(.0006)$ & $0.74(0.62-0.87)$ & $0.79(0.66-0.94)$ & .0064 \\
\hline & Chemo $=$ Rad & 447 & $34(<.0001)$ & $0.72(0.62-0.83)$ & $0.77(0.67-0.9)$ & .0006 \\
\hline
\end{tabular}

CI, Confidence interval; Chemo, chemotherapy; Rad, radiation; Chemo + Rad, sequential chemotherapy followed by radiation; Rad + Chemo, sequential radiation followed by chemotherapy; Chemo $=$ Rad, concurrent chemoradiation therapy.

TABLE E11. Analysis of stage group 4, margin-negative patients by pN stage (N0/N1 vs N2)

\begin{tabular}{|c|c|c|c|c|c|c|}
\hline \multirow[b]{3}{*}{ pN stage } & \multirow[b]{3}{*}{ Postoperative treatment } & \multirow[b]{3}{*}{$\mathbf{n}$} & \multicolumn{4}{|c|}{ Stage group 4: stage IIIA (T1-3, N2; T3, N1) } \\
\hline & & & \multicolumn{4}{|c|}{ Margin-negative patients only (R0) } \\
\hline & & & $\begin{array}{c}\text { 5-y overall } \\
\text { survival (log-rank } \\
P \text { value) }{ }^{*} \\
\end{array}$ & $\begin{array}{c}\text { Unadjusted } \\
\text { hazard ratio }(95 \% \mathrm{CI})\end{array}$ & $\begin{array}{c}\text { Adjusted hazard } \\
\text { ratio }(95 \% \mathrm{CI}) \\
\end{array}$ & $\begin{array}{c}\text { Propensity-adjusted } \\
\text { hazard ratio }(95 \% \mathrm{CI})\end{array}$ \\
\hline \multirow[t]{7}{*}{ N0,N1 } & No treatment & 396 & 27 (Referent) & 1.00 (Referent) & 1.00 (Referent) & 1.00 (Referent) \\
\hline & Chemo only & 464 & $46(<.0001)$ & $0.46(0.37-0.57)$ & $0.49(0.39-0.62)$ & $2.54(1.30-4.95)$ \\
\hline & Radiation only & 35 & $28(.6906)$ & $1.10(0.71-1.70)$ & $0.94(0.59-1.48)$ & $1.08(0.69-1.69)$ \\
\hline & Chemo + Rad & 137 & $23(.2422)$ & $0.85(0.65-1.10)$ & $0.89(0.67-1.18)$ & $0.92(0.70-1.21)$ \\
\hline & Chemo + Rad & 74 & $23(.1490)$ & $0.77(0.54-1.09)$ & $0.85(0.59-1.21)$ & $0.84(0.59-1.19)$ \\
\hline & $\operatorname{Rad}+$ Chemo & 36 & $12(.5019)$ & $1.28(0.79-2.08)$ & $1.28(0.77-2.13)$ & $1.11(0.67-1.84)$ \\
\hline & Chemo $=\operatorname{Rad}$ & 27 & $29(.3696)$ & $0.75(0.47-1.22)$ & $0.73(0.44-1.23)$ & $0.96(0.58-1.59)$ \\
\hline \multirow[t]{7}{*}{$\mathrm{N} 2$} & No treatment & 1723 & 23 (Referent) & 1.00 (Referent) & 1.00 (Referent) & 1.00 (Referent) \\
\hline & Chemo only & 2056 & $38(<.0001)$ & $0.62(0.56-0.68)$ & $0.65(0.59-0.71)$ & $0.65(0.59-0.72)$ \\
\hline & Radiation only & 213 & $15(.0620)$ & $1.20(1.00-1.44)$ & $1.18(0.98-1.42)$ & $1.19(0.99-1.43)$ \\
\hline & Chemo + Rad & 1722 & $40(<.0001)$ & $0.61(0.56-0.68)$ & $0.67(0.60-0.74)$ & $0.69(0.62-0.76)$ \\
\hline & Chemo + Rad & 1022 & $43(<.0001)$ & $0.55(0.49-0.62)$ & $0.61(0.54-0.69)$ & $0.61(0.54-0.69)$ \\
\hline & $\operatorname{Rad}+$ Chemo & 280 & $36(.0002)$ & $0.70(0.59-0.84)$ & $0.74(0.61-0.89)$ & $0.74(0.62-0.90)$ \\
\hline & Chemo $=\operatorname{Rad}$ & 420 & $34(<.0001)$ & $0.72(0.62-0.84)$ & $0.77(0.66-0.91)$ & $0.77(0.66-0.91)$ \\
\hline
\end{tabular}

CI, Confidence interval; Chemo, chemotherapy; Rad, radiation; Chemo + Rad, sequential chemotherapy followed by radiation; Rad + Chemo, sequential radiation followed by chemotherapy; Chemo $=R a d$, concurrent chemoradiation therapy. $* P$ values compare each treatment to referent (no treatment). 